\title{
New Adducts of Lapachol with Primary Amines
}

\author{
Mirelly D. F. Santos, José T. Litivack-Junior, Roberto V. Antunes, Tania M. S. Silva and \\ Celso A. Camara* \\ Departamento de Química, Universidade Federal Rural de Pernambuco, Campus Dois Irmãos, \\ 52171-900 Recife-PE, Brazil
}

\begin{abstract}
A reação do lapachol à temperatura ambiente, com aminas alifáticas primárias forneceu novos adutos identificados como derivados do núcleo fenazina. Os produtos foram obtidos em rendimentos bons a razoáveis (52 a 88\%), a temperatura ambiente e sem o uso de solvente, a partir da reação com alquilaminas funcionalizadas, como n-butilamina, etanolamina, 3-propanolamina, 2-metoxi-etilamina, 3-metoxi-propilamina e 2-feniletilamina.
\end{abstract}

New adducts of lapachol with neat primary aliphatic amines were obtained in a solvent-free reaction in good to reasonable yields (52 to $88 \%$ ), at room temperature. The new compounds containing a phenazine moiety were obtained from suitable functionalized aminoalkyl compounds, including ethanolamine, 3-propanolamine, 2-methoxy-ethylamine, 3-methoxy-propylamine, n-butylamine and 2-phenetylamine.

Keywords: phenazines, lapachol, nitrogen adducts, quinones, 1,4-naphthoquinone

\section{Introduction}

Molecules containing a quinone moiety as a structural component constitute an important class of compounds in organic chemistry. The conjugated 1,4-dicarbonyl or 1,2-dicarbonyl moiety is responsible for a characteristic reactivity behavior, yielding a somewhat particular chemistry repertoire for these compounds. These functional groups are also involved in numerous biological activities, mostly cytotoxic ones. The wide range of biological activities includes anticancer, ${ }^{1}$ inhibitors of topoisomerase $\mathrm{II},{ }^{2}$ highlighting their use as valuable candidates in neglected tropical diseases, including leishmaniasis, ${ }^{3}$ tuberculosis ${ }^{4}$ and tripanosomiasis. ${ }^{5}$ Among the naturally occurring naphthoquinones in Tabebuia spp, lapachol, $\alpha$-lapachone and $\beta$-lapachone and xyloidones are the most abundant and studied compounds in these and related species. ${ }^{6} \beta$-Lapachone, in particular, showed a more defined and impressive biological profile in antitumor screenings than the regioisomer $\alpha$-lapachone or lapachol. ${ }^{7,8}$ The molecular mechanism involved in the observed antitumoral and cytotoxic activities of these compounds seems to be mainly related to the ability of the quinone nucleus in participating in redox processes,

*e-mail: ccelso@dq.ufrpe.br in which the cascade of one-electron radical enzymaticmediated reactions results in the formation of superoxide radical anions, responsible for cellular damage in living media. ${ }^{9}$ In a continuing work concerning the reactivity of lapachol and related quinones, ${ }^{10,11}$ we now present our results concerning the reaction of lapachol with primary aliphatic amines. This smooth reaction conduced to new adducts with a cyclic structure consisting of a phenazine nucleus. ${ }^{12}$ Our initial observation, unlike previous reports, ${ }^{13}$ is that lapachol $\mathbf{1}$ is almost entirely consumed when mixed with primary amines, like n-butylamine, ethanolamine, 3-hydroxypropylamine, 2-methoxyethylamine, 3-methoxypropylamine, and 2-phenylethylamine. The reaction of some few secondary cyclic amines with lapachol have also been described previously, with some unusual spectroscopic data found..$^{14}$

\section{Results and Discussion}

The products were formed in reactions at room temperature, using solvent-free conditions with neat amines, proceeding slowly within $24-48 \mathrm{~h}$ to give polar dye compounds. These presented as crystals with brilliant green color when in solid form, and deep-blue when in solution with polar solvents. These compounds were identified by usual spectroscopic methods as presenting the structure 
2a-f shown, as an adduct of two molecules of lapachol with the corresponding amine (Scheme 1). The synthesis of compounds containing this type of phenazine nucleus was described previously for the reactions with aromatic amines. ${ }^{12}$ The previously described conditions in the cited literature includes thermal treatment of the reaction mixtures. The present work extends this methodology to a more environmentally benign procedure, with reasonable to good yields (52-88\%) of products in good purity, with easy isolation and reproducibility. The reaction was conducted in an open vessel, based on the experimental observation that the reaction proceeds faster in the presence of molecular oxygen. A closed sample in inert gas remains a blue liquid for a very long time, and, when opened, an instant precipitation of green crystals is observed in the flask. The procedure of isolation consists in filtering the formed crystals at vacuum followed by water washing with some methanol added. The crystals obtained are sufficiently pure to TLC inspection and spectrometric analysis. Attempts to recrystalize in different solvent mixtures results in decomposition of the starting material, as observed by TLC. Although the detailed mechanism for this reaction remains to be further clarified, a lot of precedent data is available to clarify some points. The nucleophilic addition of amines to carbonyl of quinone nucleus is a known reaction, although the subsequent reactions of the formed adducts are characteristic of quinone chemistry. ${ }^{15}$ These hydroxyquinone adducts on the carbonyl with amines can undergo a reaction called Strecker degradation, ${ }^{16}$ rather than the conventional Schiff's base addition followed by water elimination product. These alternative path probably consists in an imine-enamine isomerization, followed by hydrolysis in situ with formal dealkylation of the primary amine adduct. Recently the reaction of 2-hydroxy-1,4naphthoquinone (lawsone) with primary amino acids was described as a new class of fingermark detection reagents. ${ }^{15}$ It is noteworthy that in the cited work the best profile for fingermark detection was achieved with the amino acid lysine. On the basis of our findings, we believe that this is due to the presence of a more nucleophylic amino alkyl side chain residue. The authors in this work did not support the proposed adducts with any kind of experimental data; no attempts for isolation were made or spectroscopic characterization of products furnished. Additionally, the fluorescence emission spectra of some of the adducts reported here are in agreement with the profile previously reported by Jelly et al. ${ }^{15}$ (see supplementary information, SI, Figure S25). However, in opposition with the structure furnished by Jelly et al., ${ }^{15}$ the elemental microanalysis is consistent with the presence of two nitrogen atoms (see Experimental section), with just one alkyl residue from the amine, supporting a phenazine nucleus. Another observation concerns the extremely low reactivities of amino acids as glycine and phenylalanine. These amino acids, even in the presence of some inorganic and organic basis, reacts very slowly furnishing complex mixtures at TLC inspection. We also were unable to isolate some useful products from the reaction of lapachol with benzylamine. At the same conditions listed above, the reaction proceeds rapidly with a prolific profile of by-products, and attempts to purification by column chromatography were also unsuccessful. A proposed mechanism for the formation of (2) from (1) is presented in Figure 1. The characterization of 2a by ${ }^{1} \mathrm{H}$ and ${ }^{13} \mathrm{C}$ NMR analysis showed a simplified spectra, attributed to the assumption of canonical equivalent resonance forms on the proposed structure (Figure 1). A singlet at $1.79 \mathrm{ppm}(6 \mathrm{H})$ and a doublet at $1.62 \mathrm{ppm}(0.5 \mathrm{~Hz}$, $6 \mathrm{H})$ were attributed to methyl signals of the prenyl side chain. The methyl groups are coupled with the vinylic proton at $5.31 \mathrm{ppm}(\mathrm{m}, 2 \mathrm{H})$, and the vinylic proton coupled with the allylic methylene which appear at $3.47 \mathrm{ppm}(\mathrm{m}$, $4 \mathrm{H}, 7 \mathrm{~Hz})$. The aromatic protons are two double doublets at $8.69 \mathrm{ppm}(2 \mathrm{H}, 8 / 0.5 \mathrm{~Hz})$ and $8.16 \mathrm{ppm}(2 \mathrm{H}, 8 / 0.5 \mathrm{~Hz})$, attributed to the ortho hydrogens relative to quinone/ phenazine nucleus. The other two meta hydrogens absorbs as a double of double doublets at $7.60(2 \mathrm{H}, 0.5 / 1 / 8 \mathrm{~Hz})$ and $7.40 \mathrm{ppm}(2 \mathrm{H}, 0.5 / 1 / 8 \mathrm{~Hz})$. The methylene attached to nitrogen appears as a triplet at $2.84 \mathrm{ppm}(2 \mathrm{H}, 5.5 \mathrm{~Hz})$, and couples with another carbynolic methylene as a triplet at $3.56 \mathrm{ppm}(2 \mathrm{H}, 5.5 \mathrm{~Hz})$.

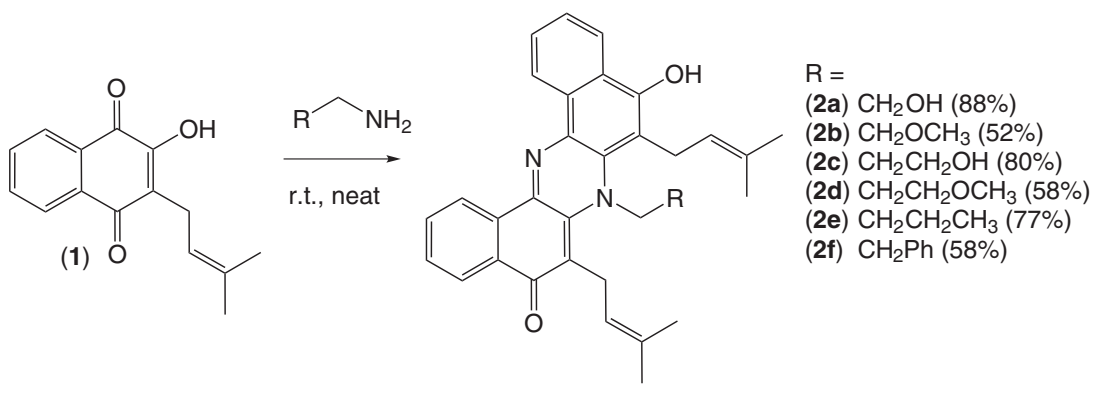

Scheme 1. Reaction of lapachol adducts with primary neat alkyl amines. 


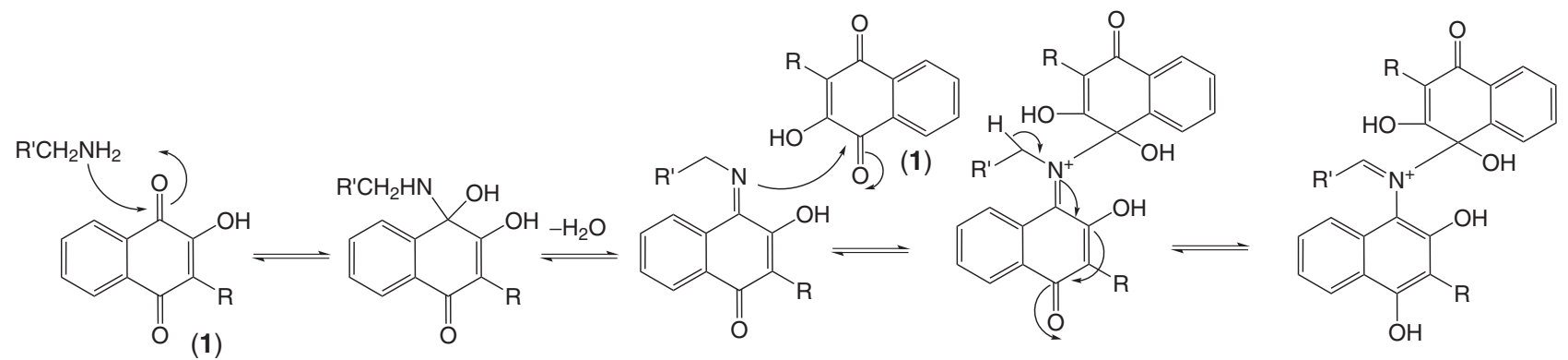

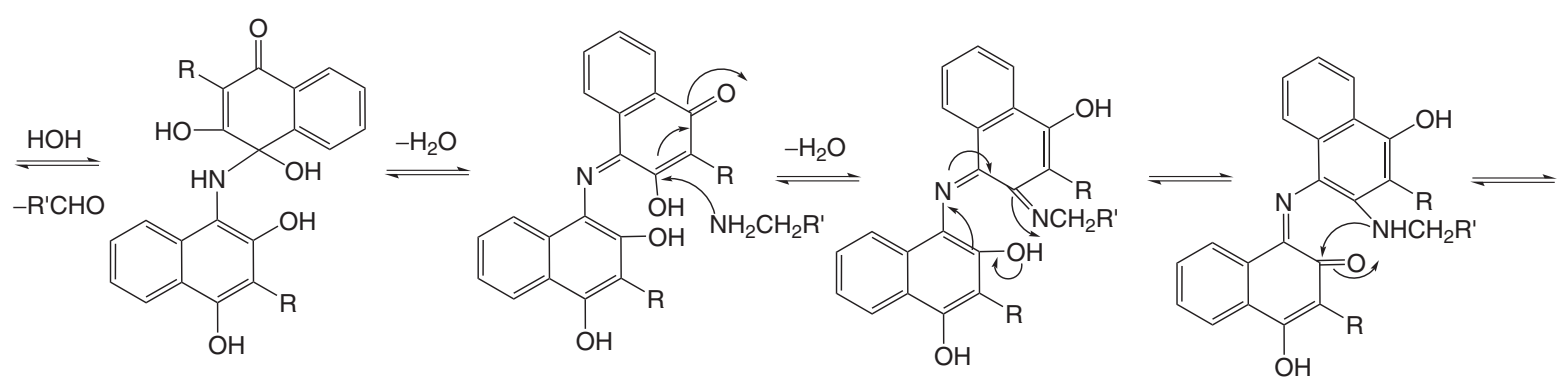

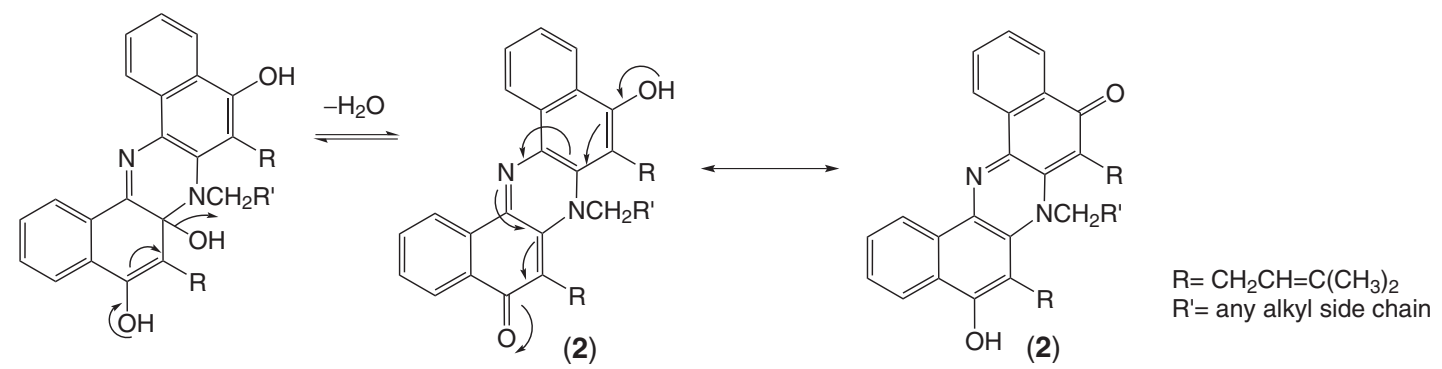

Figure 1. A proposed mechanism for the formation of (2) from (1) and the tautomerism in phenazine adducts (2)

\section{Conclusions}

In the present study, we presented a new, simple and solvent-free procedure for the reaction of lapachol with primary amines at ambient temperature. The obtained yields were good to reasonable and reproducible, furnishing new derivatives of phenazines from lapachol, with suitable functionalized amines.

\section{Experimental}

\section{General}

Unless otherwise stated, all common reagents and solvents were used as obtained from commercial suppliers without further purification. Lapachol was previously obtained by usual methods. Melting points were obtained from an electrically heated metal block apparatus (Quimis), and were not corrected. FTIR spectra were obtained in a Bomen-Michelson spectrophotometer using $\mathrm{KBr}$ film. NMR spectra were recorded in a Varian-Mercury $200 \mathrm{MHz}$ for ${ }^{1} \mathrm{H}$ and $50.3 \mathrm{MHz}$ for ${ }^{13} \mathrm{C}$, with DMSO- $d_{6}$ as solvents, and HR mass spectra, on a VG Autospec spectrometer (electron-impact at $70 \mathrm{eV}$ ). The reaction progress was monitored using thin layer chromatography on a silicagel UV254 TLC aluminum sheet. The elemental analysis was performed on a CE EA1110 instrument at the Central Analítica (Departamento de Química Fundamental Universidade Federal de Pernambuco).

\section{General procedure for the synthesis of adducts $2 a-f$}

A solution of lapachol 1 (0.5 g) and corresponding amine $(1 \mathrm{~mL})$ was stirred in an open flask, for a $48 \mathrm{~h}$ period at room tempertaure. The initially yellow solution turned to deep red, and finally to a greenish-blue precipitated material, or deep-blue solution with crystals was obtained. TLC inspection of the reaction mixture showed an almost complete consumption of lapachol after $48 \mathrm{~h}$. With the aid of water with some methanol (5\%), the solids were filtered in vacuum, washed with additional water, dried at room temperature as fluorescent green or deep blue crystals. 
9-Hydroxy-7-(2-hydroxyethyl)-6,8-bis(3-methylbut2-enyl)dibenzo[a,j]phenazin-5(7H)-one (2a): $88 \%$ yield, deep blue crystals with mp $281-3{ }^{\circ} \mathrm{C}$; IR $(\mathrm{KBr}) v_{\max } / \mathrm{cm}^{-1}$ : 3200, 3058, 2962, 2910, 2852, 1608, 1570, 1508, 1409, $1126,1028,789$; UV (EtOH) $\lambda_{\max } / \mathrm{nm}: 650,627,528,287$; ${ }^{1} \mathrm{H}$ NMR (DMSO- $\left.d_{6}, 200 \mathrm{MHz}\right) \delta 1.62(\mathrm{~s}, 6 \mathrm{H}), 1.79$ (s, $6 \mathrm{H}), 2.84(\mathrm{t}, 2 \mathrm{H}, J 5.5 \mathrm{~Hz}), 3.47(\mathrm{~m}, 4 \mathrm{H}, J 7 \mathrm{~Hz}), 3.56(\mathrm{t}$, $2 \mathrm{H}, J 5.5 \mathrm{~Hz}), 5.31(\mathrm{~m}, 2 \mathrm{H}), 7.40(\mathrm{ddd}, 2 \mathrm{H}, J 7 / 1 / 1 \mathrm{~Hz})$, 7.60 (ddd, $2 \mathrm{H}, J 7 / 1 / 1 \mathrm{~Hz}$ ), 8.16 (dd, $2 \mathrm{H}, J 8 / 0.5 \mathrm{~Hz}$ ), 8.69 (dd, $2 \mathrm{H}, J 8 / 0.5 \mathrm{~Hz}) ;{ }^{13} \mathrm{C}$ NMR (APT, DMSO- $d_{6}, 50 \mathrm{MHz}$ ) $\delta 17.9,21.7,25.5,41.2,57.5,111.2,121.7,122.0,123.5$, 124.9, 128.7, 129.3, 130.0, 131.7, 148.4, 176.8. Elemental analysis for $\mathrm{C}_{32} \mathrm{H}_{32} \mathrm{~N}_{2} \mathrm{O}_{3} \cdot \mathrm{H}_{2} \mathrm{O}$ found: $\mathrm{C}, 72.89 ; \mathrm{H}, 7.39 ; \mathrm{N}$, 5.41; Requires: C, 72.70; H 6.86; N, 5.30\%.

9-Hydroxy-7-(2-methoxyethyl)-6,8-bis(3-methylbut2-enyl)dibenzo[a,j]phenazin-5(7H)-one ( $2 \mathrm{~b})$ : $52 \%$ yield, greenish blue crystals with mp $206-8^{\circ} \mathrm{C}$; IR $(\mathrm{KBr}) v_{\max } / \mathrm{cm}^{-1}$. 3360, 2920, 2910, 1654, 1570, 1508, 1485, 1115, 759; UV (EtOH) $\lambda_{\max } / \mathrm{nm}: 648,622,522,280 ;{ }^{1} \mathrm{H}$ NMR (DMSO- $d_{6}$, $200 \mathrm{MHz}) \delta 1.62$ (s, 6H), 1.69 (s, 6H), $2.96(\mathrm{~m}, 2 \mathrm{H}), 3.25$ (s, 3H), $3.48(\mathrm{~m}, 4 \mathrm{H}), 5.31(\mathrm{~m}, 2 \mathrm{H}), 7.42(\mathrm{dd}, 2 \mathrm{H}, J 7 / 7.8$ $\mathrm{Hz}), 7.61$ (dd, 2H, $J 7 / 7.8 \mathrm{~Hz}), 8.18$ (d, 2H, J 7.8Hz), 8.70 (d, $2 \mathrm{H}, J 7.8 \mathrm{~Hz}$ ); ${ }^{13} \mathrm{C}$ NMR (APT, DMSO- $\left.d_{6}, 50 \mathrm{MHz}\right) \delta$ 17.9, 21.8, 25.5, 58.1, 68.2, 111.3, 121.7, 122.2, 123.5, 125.2, 128.7, 129.3, 130.1, 131.7, 148.4, 176.6; Elemental analysis for $\mathrm{C}_{33} \mathrm{H}_{34} \mathrm{~N}_{2} \mathrm{O}_{3} .3 \mathrm{H}_{2} \mathrm{O}$ found: $\mathrm{C}, 69.61 ; \mathrm{H}, 6.67 ; \mathrm{N}$, 4.14; Requires C, 70.44; H, 6.67; N, 4.98\%.

9-Hydroxy-7-(3-hydroxypropyl)-6,8-bis(3-methylbut-2enyl)dibenzo[a,j]phenazin-5(7H)-one (2c): $84 \%$ yield as a greenish blue solid with mp $251-3^{\circ} \mathrm{C}$; IR (KBr) $v_{\max } / \mathrm{cm}^{-1}$ : 3414, 2924, 1620, 1570, 1485, 1458, 1100, 779; UV (EtOH) $\lambda_{\max } / \mathrm{nm}: 650,622,528,287 ;{ }^{1} \mathrm{H}$ NMR (DMSO- $d_{6}, 200$ MHz) $\delta 1.55,(\mathrm{~s}, 6 \mathrm{H}), 1.62(\mathrm{~m}, 2 \mathrm{H}), 1.72(\mathrm{~s}, 6 \mathrm{H}), 2.79(\mathrm{t}$, $2 \mathrm{H}, J 7 \mathrm{~Hz}), 3.42(\mathrm{~m}, 6 \mathrm{H}), 5.24(\mathrm{~m}, 2 \mathrm{H}), 7.35(\mathrm{ddd}, 2 \mathrm{H}$, $J$ 1/8/8 Hz), 7.54 (ddd, 2H, $J$ 1/8/8 Hz), 8.11 (d, 2H, $J 8$ $\mathrm{Hz}$ ), $8.63(\mathrm{~d}, 2 \mathrm{H}, J 8 \mathrm{~Hz}) ;{ }^{13} \mathrm{C}$ NMR (APT, DMSO- $d_{6}, 50$ MHz) $\delta 17.9,21.8,25.5,30.1,36.9,58.0,111.4,121.8$, 122.4, 123.5, 124.9, 125.1, 128.7, 129.3, 130.1, 131.7, 148.4, 176.6; Elemental analysis for $\mathrm{C}_{33} \mathrm{H}_{36} \mathrm{~N}_{2} \mathrm{O}_{4} \cdot \mathrm{H}_{2} \mathrm{O}$ found: C, 72.90; H, 7.40; N, 5.00; Requires: C, 73.04; H, $7.06 ; \mathrm{N}, 5.16 \%$.

9-Hydroxy-7-(3-methoxypropyl)-6,8-bis(3-methylbut-2enyl)dibenzo[a,j]phenazin-5(7H)-one (2d): $58 \%$ yield as a greenish blue solid with mp $242-4{ }^{\circ} \mathrm{C}$; IR (KBr) $v_{\max } / \mathrm{cm}^{-1}$ : 3300, 2958, 2920, 1620, 1570, 1554, 1485, 1280, 1249, 1185, 1130; UV (EtOH) $\lambda_{\max } / \mathrm{nm}: 650,622,528,290 ;{ }^{1} \mathrm{H}$ NMR (DMSO- $\left.d_{6}, 200 \mathrm{MHz}\right) \delta 1.62(\mathrm{~s}, 6 \mathrm{H}), 1.78(\mathrm{~s}, 6 \mathrm{H})$, $1.78(\mathrm{~m}, 2 \mathrm{H}), 2.81(\mathrm{~m}, 2 \mathrm{H}), 3.19(\mathrm{~s}, 3 \mathrm{H}), 3.35(\mathrm{~m}, 2 \mathrm{H}), 3.48$ (d, 4H, J 6.6 Hz), $5.30(\mathrm{~m}, 2 \mathrm{H}), 7.41(\mathrm{dd}, 2 \mathrm{H}, \mathrm{J} 8 / 8 \mathrm{~Hz})$, 7.61 (dd, 2H, J 8/8 Hz), 8.17 (d, 2H, J 8 Hz), 8.70 (d, 2H, $\mathrm{J} 8 \mathrm{~Hz}$ ); ${ }^{13} \mathrm{C}$ NMR (APT, DMSO- $\left.d_{6}, 50 \mathrm{MHz}\right) \delta 18.0,21.8$, 25.5, 26.5, 37.0, 58.0, 68.9, 111.3, 121.7, 122.1, 123.5, 125.0, 128.8, 129.4, 130.1, 131.8, 148.5, 176.9; Elemental analysis for $\mathrm{C}_{34} \mathrm{H}_{38} \mathrm{~N}_{2} \mathrm{O}_{4} \cdot 2 \mathrm{H}_{2} \mathrm{O}$ found: $\mathrm{C}, 71.18 ; \mathrm{H}, 7.61 ; \mathrm{N}$, 5.02; Requires: C, 71.06; H, 7.37; N, 4.87\%.

7-Butyl-9-hydroxy-6,8-bis(3-methylbut-2-enyl) dibenzo[a,j]phenazin-5(7H)-one (2e): $80 \%$ yield of a greenish blue solid with mp $276-9{ }^{\circ} \mathrm{C}$; IR (KBr) $v_{\max } / \mathrm{cm}^{-1}$ : 3400, 2920, 2854, 1585, 1508, 1477, 1454, 1415, 1300, 1249, 1157, 1118; UV (EtOH) $\lambda_{\max } / \mathrm{nm}: 650,622,524$, 284, 250, 245; ${ }^{1} \mathrm{H}$ NMR (DMSO- $\left.d_{6}, 200 \mathrm{MHz}\right) \delta 0.84(\mathrm{t}$, $3 \mathrm{H}, J 7 \mathrm{~Hz}), 1.30(\mathrm{~m}, 2 \mathrm{H}), 1.48(\mathrm{~m}, 2 \mathrm{H}), 1.63(\mathrm{~s}, 6 \mathrm{H}), 1.79$ (s, 6H), $2.76(\mathrm{t}, 2 \mathrm{H}, J 7 \mathrm{~Hz}), 3.48(\mathrm{~m}, 4 \mathrm{H}), 5.31(\mathrm{~m}, 2 \mathrm{H})$, 7.41 (dd, 2H, $J 6.8 / 6.2 \mathrm{~Hz}), 7.61(\mathrm{dd}, 2 \mathrm{H}, J 6.8 / 6.2 \mathrm{~Hz}$ ), $8.18(\mathrm{~d}, 2 \mathrm{H}, J 6.8 \mathrm{~Hz}), 8.70(\mathrm{~d}, 2 \mathrm{H}, J 6.8 \mathrm{~Hz}) ;{ }^{13} \mathrm{C} \mathrm{NMR}$ (APT, DMSO- $\left.d_{6}, 50 \mathrm{MHz}\right) \delta 13.4,17.9,19.1,21.8,25.5$, 29.1, 38.6, 111.2, 121.7, 122.1, 123.5, 124.9, 124.9, 128.7, 129.3, 130.0, 131.7, 148.4, 176.8; Elemental analysis for $\mathrm{C}_{34} \mathrm{H}_{38} \mathrm{~N}_{2} \mathrm{O}_{3} \cdot \mathrm{H}_{2} \mathrm{O}$ found $\mathrm{C}, 75.81 ; \mathrm{H}, 7.87 ; \mathrm{N}, 5.14$; Requires: C, 75.53; H, 7.46; N, 5.18\%.

9-Hydroxy-6,8-bis(3-methylbut-2-enyl)-7phenethyldibenzo[a,j]phenazin-5(7H)-one (2f): $62 \%$ of a greenish blue crystals with mp $217-9^{\circ} \mathrm{C}$; IR $(\mathrm{KBr}) v_{\text {max }} / \mathrm{cm}^{-1}$ : 2890, 2920, 1564, 1509, 1474, 1374, 1299, 1250, 1161, 1128; UV (EtOH) $\lambda_{\max } / \mathrm{nm}: 650,622,524,284,250 ;{ }^{1} \mathrm{H}$ NMR (DMSO- $\left.d_{6}, 200 \mathrm{MHz}\right) \delta 1.61(\mathrm{~s}, 6 \mathrm{H}), 1.78(\mathrm{~s}, 6 \mathrm{H})$, $2.83(\mathrm{dd}, 2 \mathrm{H}, J 6.2 / 5.4 \mathrm{~Hz}), 3.03$ (dd, $2 \mathrm{H}, J 6.2 / 5.4 \mathrm{~Hz}), 3.47$ (d, 4H, J 6.2 Hz), 5.30 (m, 2H), 7.28 (m, 5H), 7.40 (ddd, $2 \mathrm{H}, J 1.2 / 6.8 / 8.2 \mathrm{~Hz}$ ), 7.61 (ddd, $2 \mathrm{H}, J 1.2 / 6.8 / 8.2 \mathrm{~Hz}$ ), $8.16(\mathrm{dd}, 2 \mathrm{H}, J 1.2 / 6.8 \mathrm{~Hz}), 8.69(\mathrm{dd}, 2 \mathrm{H}, J 1.2 / 6.8 \mathrm{~Hz}) ;{ }^{13} \mathrm{C}$ NMR (APT, DMSO- $\left.d_{6}, 50 \mathrm{MHz}\right) \delta 17.9,21.8,25.5,36.2$, 38.2, 111.4, 121.7, 122.4, 123.4, 124.9, 125.0, 126.7, 128.1, 128.3, 128.4, 128.6, 128.7, 128.8, 128.2, 130.1, 131.7, 148.3, 178.5; Elemental analysis for $\mathrm{C}_{38} \mathrm{H}_{38} \mathrm{~N}_{2} \mathrm{O}_{3} .2 \mathrm{H}_{2} \mathrm{O}$ found: C, 74.84; H, 7.16; N, 4.30; Requires: C, 75.22; H, $6.98 ; \mathrm{N}, 4.62 \%$.

\section{Supporting Information}

Supplementary information (Figures S1-S25) is available free of charge at http://jbcs.sbq.org.br, as a PDF file.

\section{Acknowledgments}

We are very grateful to Dr. Eduardo Oliveira (LTFUFPB) for the critical revision of the original manuscript 
and useful suggestions. Finnancial support from the Coordenação de Aperfeiçoamento do Pessoal de Nível Superior (CAPES), and Fundação de Amparo à Pesquisa do Estado de Pernambuco (FACEPE) are gratefully acknowledged. We also thank the Conselho Nacional de Desenvolvimento Científico e Tecnológico (CNPq-Brazil), and Analytical Center of LTF-UFPB, DQ-UFPE and CENAPESQ-UFRPE.

\section{References}

1. Planchon, S. M.; Wuerzberger, S.; Frydman, B.; Witiak, D. T.; Hutson, P.; Church, D. R.; Wilding, G.; Boothman, D. A.; Cancer Res. 1995, 55, 3706.

2. Krishnan, P.; Bastow, K. F.; Cancer Chemother. Pharmacol. 2001, 47, 187.

3. Fournet, A.; Angelo, A.; Munoz, V.; Roblot, F.; Hocquemiller, R.; Cava, A.; J. Ethnopharmacol. 1992, 37, 159.

4. Silva, R. S. F.; Pinto, M. D. F. R.; Goulart, M. O. F.; de Souza, J. D.; Neves, I; Lourenço, M. C. S.; Pinto, A. V.; Eur. J. Med. Chem. 2009, 44, 2334.

5. Pinto, A. V.; de Castro, S. L.; Molecules 2009, 14, 4570.

6. Hussain, H.; Krohn, K.; Ahmad, V. U.; Miana, G. A.; Green, I. R.; Arkivoc 2007, 145.

7. Li, C. J.; Averboukh, L.; Pardee, A. B.; J. Biol. Chem. 1993, 268, 22463.
8. Silva, M. N.; Ferreira, V. F.; Souza, M. C. B. V.; Quim. Nova, 2003, 26, 407.

9. Kitamura, M.; Matsuda, H.; Kimura, S.; Iyanagi, T.; Adv. Exp. Med. Biol. 2001, 500, 323.

10. Cunha, A. S.; Lima, E. L. S.; Pinto, A. C.; Esteves-Souza, A.; Echevarria, A.; Camara, C. A.; Vargas, M. D.; Torres, J. C.; J. Braz. Chem. Soc. 2006, 17, 439.

11. Esteves-Souza, A.; Figueiredo, D. V.; Camara, C. A.; Vargas, M. D.; Pinto, A. C. ; Echevarria, A.; Braz. J. Med. Biol. Res. 2007, 40, 1399.

12. Neves-Pinto, C.; Malta, V. R.; Pinto, M. C. F. R.; Santos, R. H.; De Castro, S. L.; Pinto, A. V.; J. Med. Chem. 2002, 45, 2112.

13. Chaves, J. P.; Pinto, M. C. F. R.; Pinto, A. V.; J. Braz. Chem. Soc. 1990, 1, 21.

14. Oliveira, M. F.; Lemos, T. L. G.,; Mattos, M. C.; Segundo, T. A.; Santiago, G. M. P., Braz-Filho R.; An. Acad. Bras. Cienc. 2002, 74, 211.

15. Jelly, R.; Lewis, S. W.; Lennard, C.; Lim, K. F.; Almog J.; Chem. Commun. 2008, 3513.

16. Spyroudis, S.; Molecules 2000, 5, 1291.

Submitted: July 12, 2010

Published online: January 25, 2011 


\section{Supplementary Information}

\section{New Adducts of Lapachol with Primary Amines}

\section{Mirelly D. F. Santos, José T. Litivack-Junior, Roberto V. Antunes, Tania M. S. Silva and Celso A. Camara*}

Departamento de Química, Universidade Federal Rural de Pernambuco, Campus Dois Irmãos, 52171-900 Recife-PE, Brazil

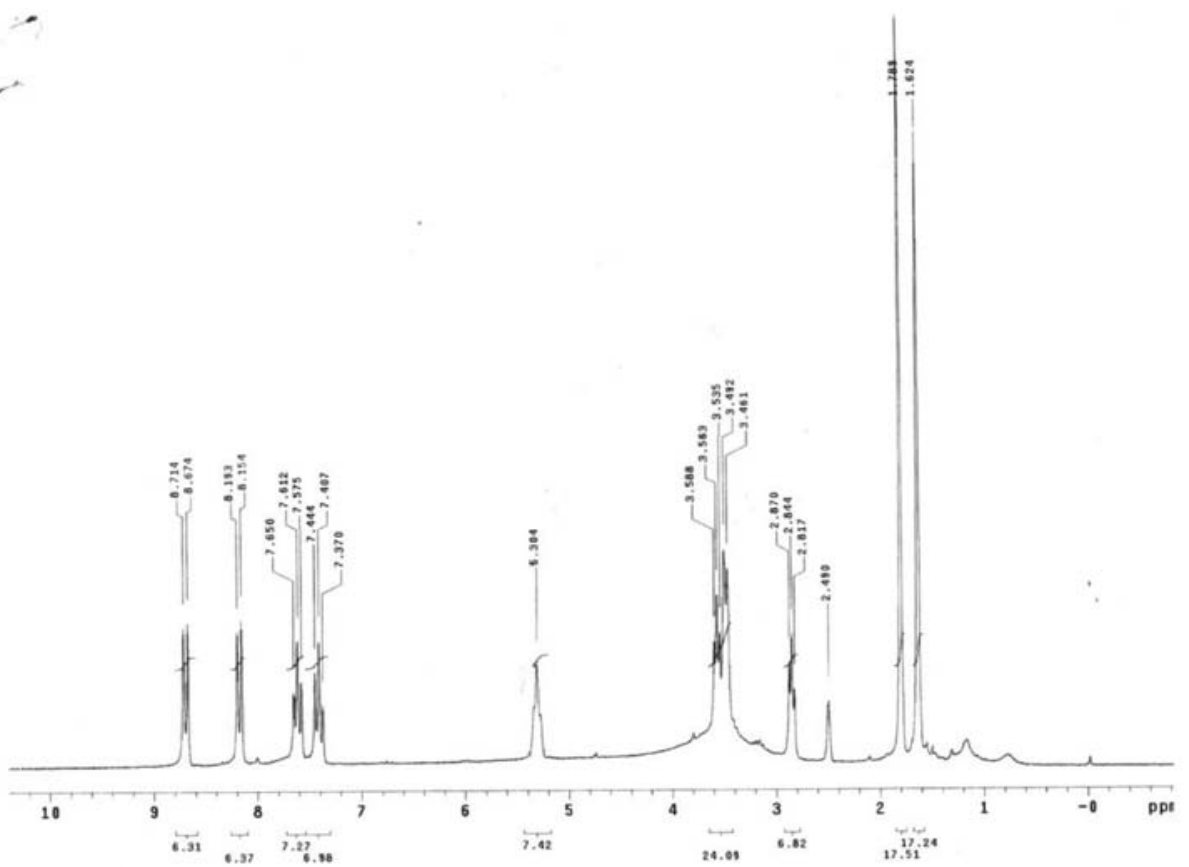

Figure S1. ${ }^{1} \mathrm{H}$ NMR spectrum (DMSO- $d_{6}, 200 \mathrm{MHz}$ ) of $\mathbf{2 a}$. 


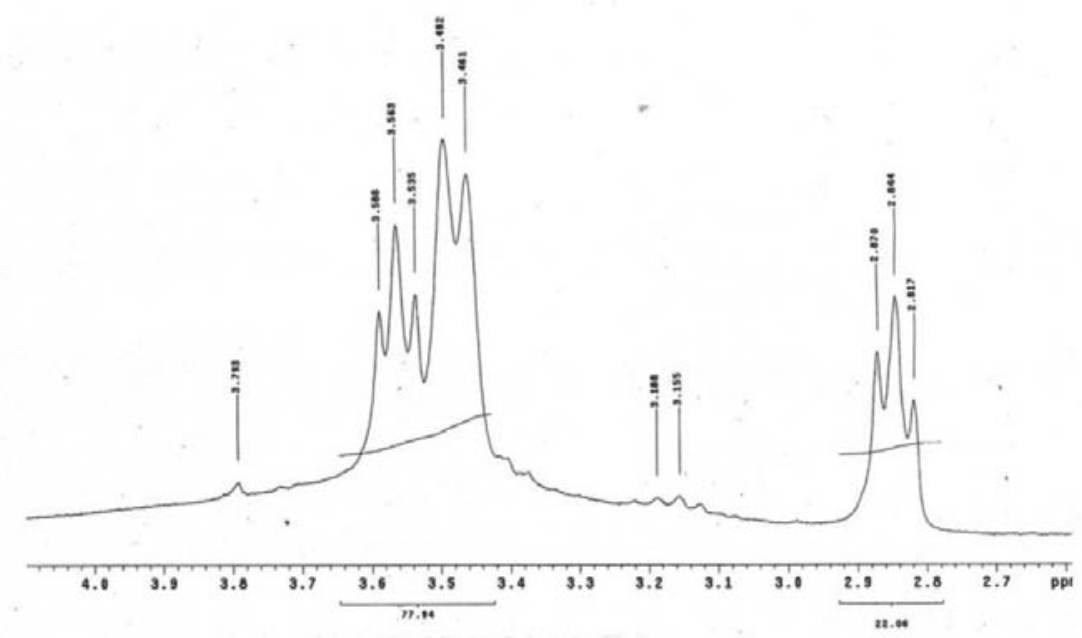

Figure S2.. ${ }^{1} \mathrm{H}$ NMR expansion spectrum (DMSO- $d_{6}, 200 \mathrm{MHz}$ ) of $2 \mathbf{a}$.
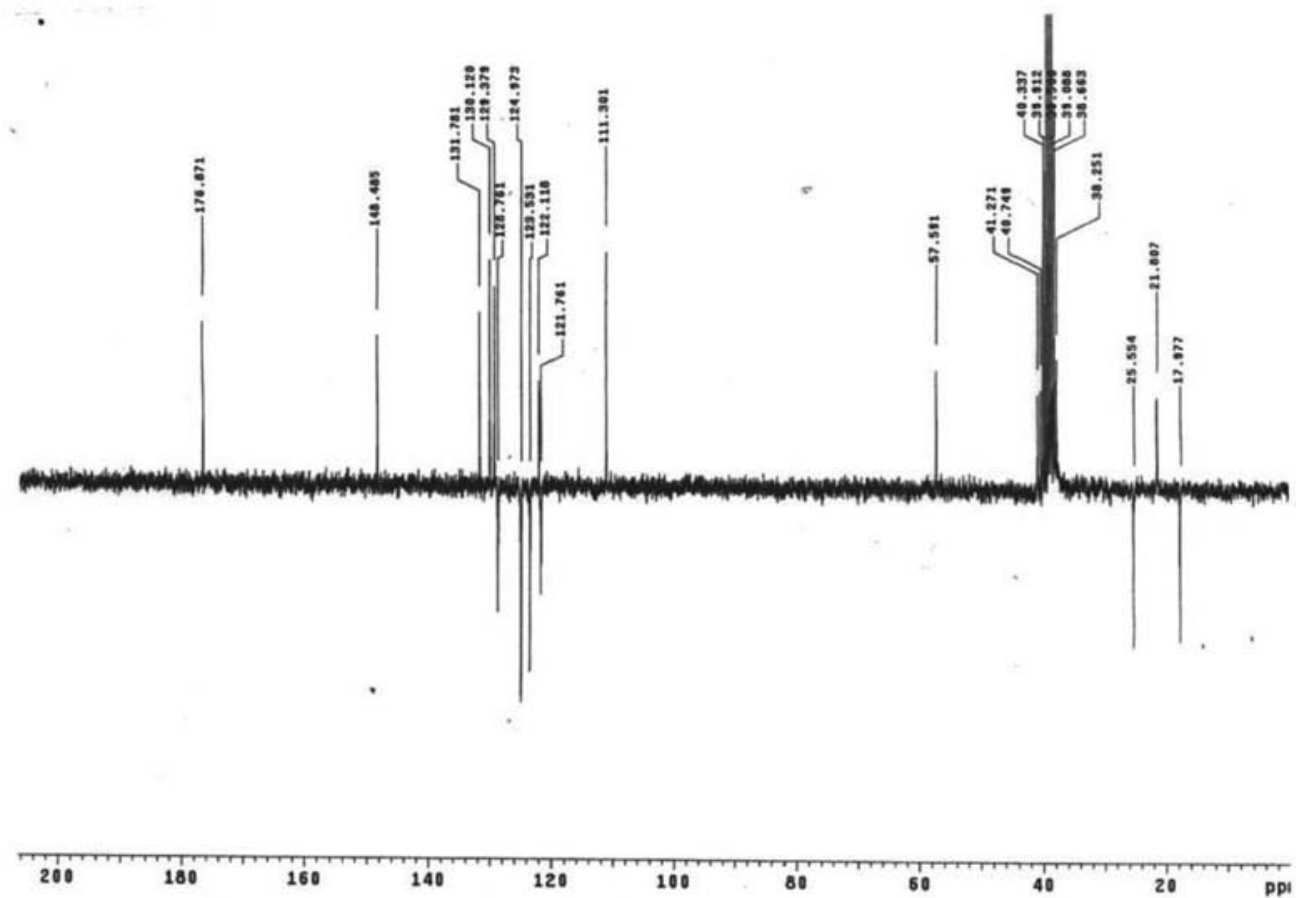

Figure S3. ${ }^{13} \mathrm{C}$ NMR (APT) spectrum (DMSO- $d_{6}, 50 \mathrm{MHz}$ ) of $2 \mathbf{2 a}$. 


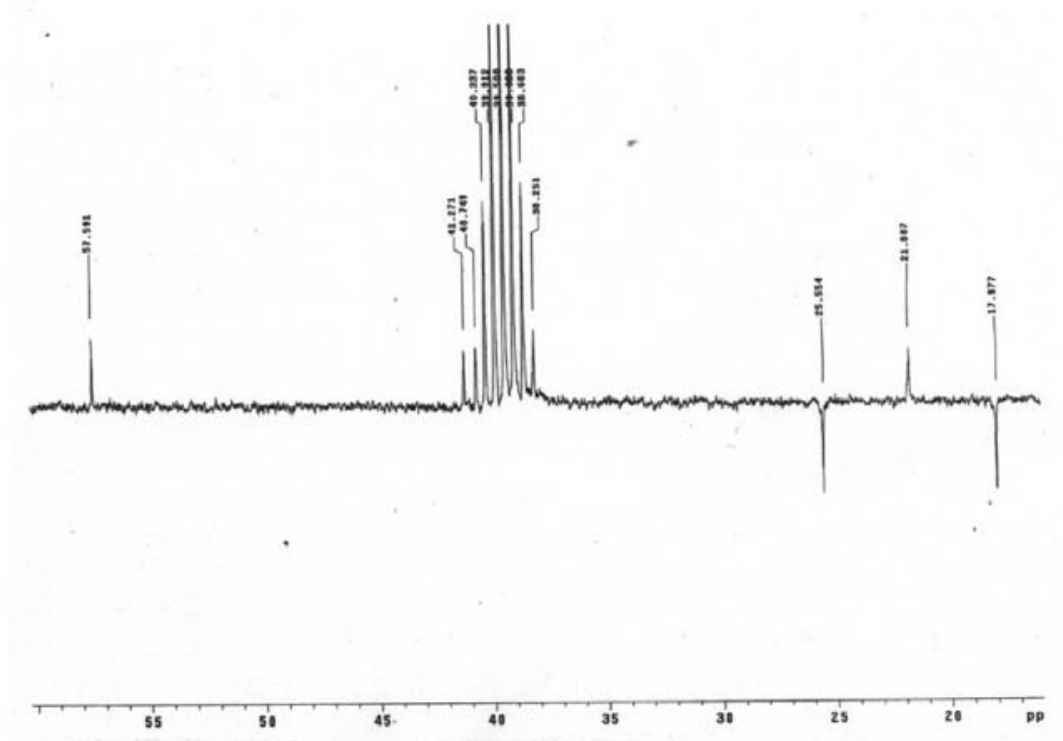

Figure S4. ${ }^{13} \mathrm{C}$ NMR (APT) spectrum expansion (DMSO- $d_{6}, 50 \mathrm{MHz}$ ) of $\mathbf{2 a}$.
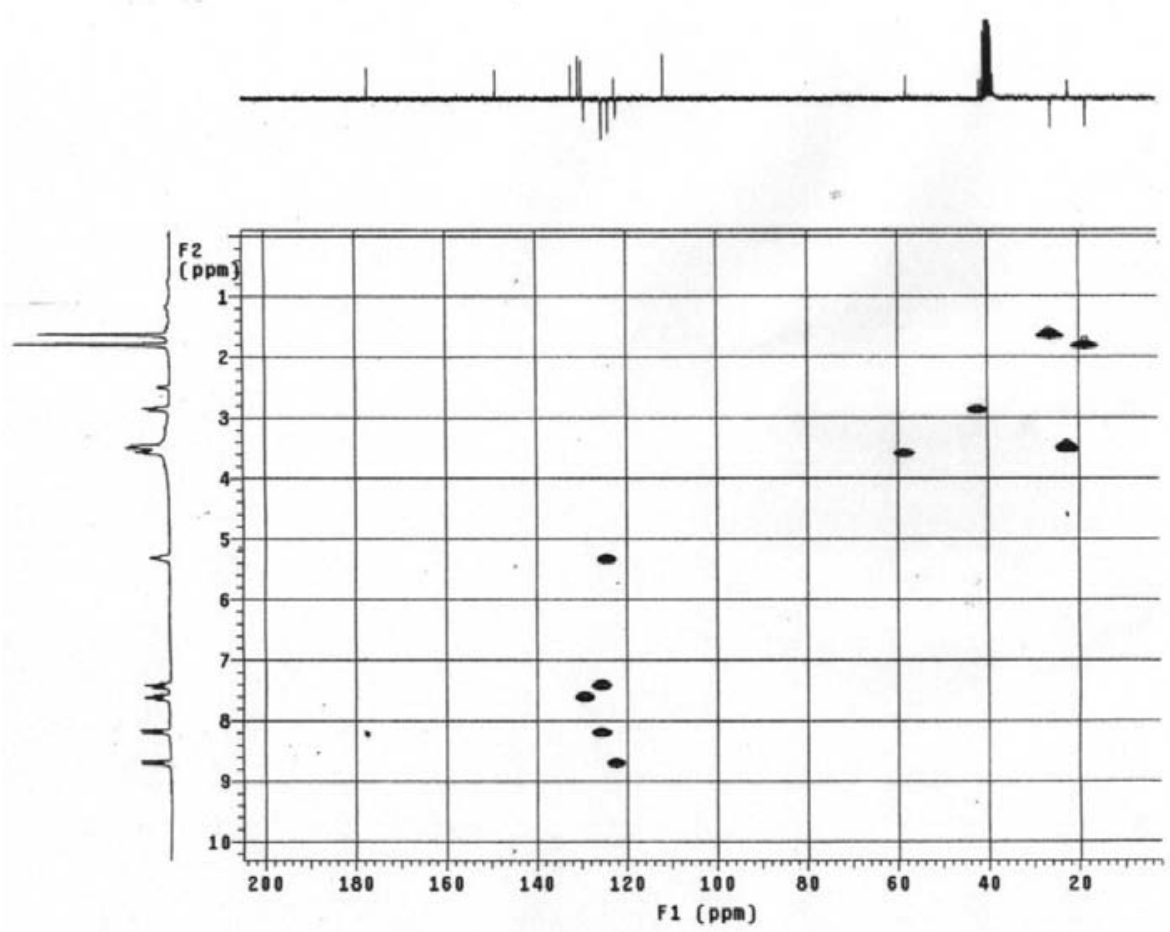

Figure S5. HMQC spectrum of 2a. 


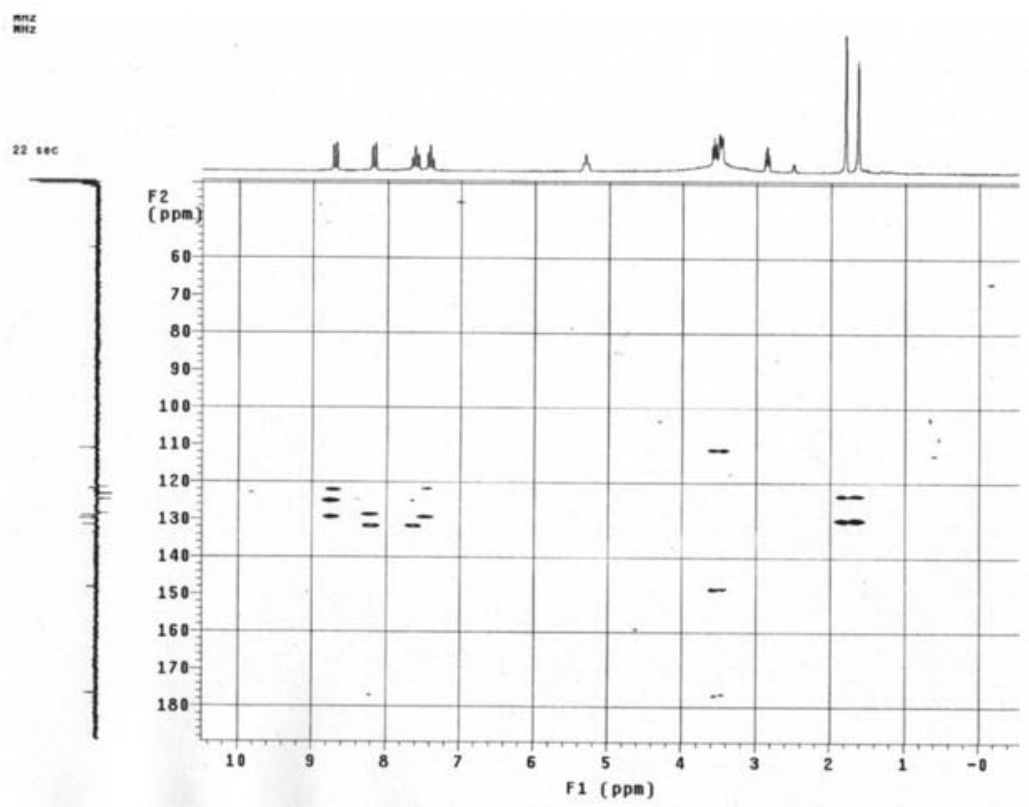

Figure S6. COLOC spectrum of $\mathbf{2 a}$.

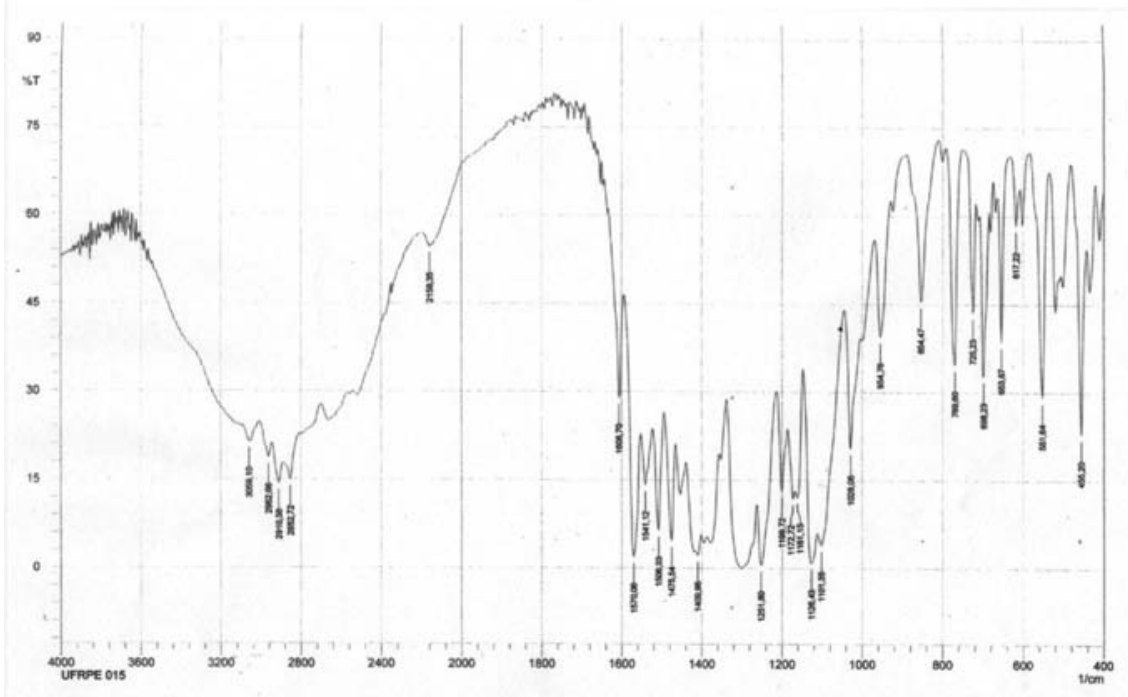

Figure S7. Infrared spectrum $\left(\mathrm{KBr}, \mathrm{cm}^{-1}\right)$ of $\mathbf{2 a}$. 


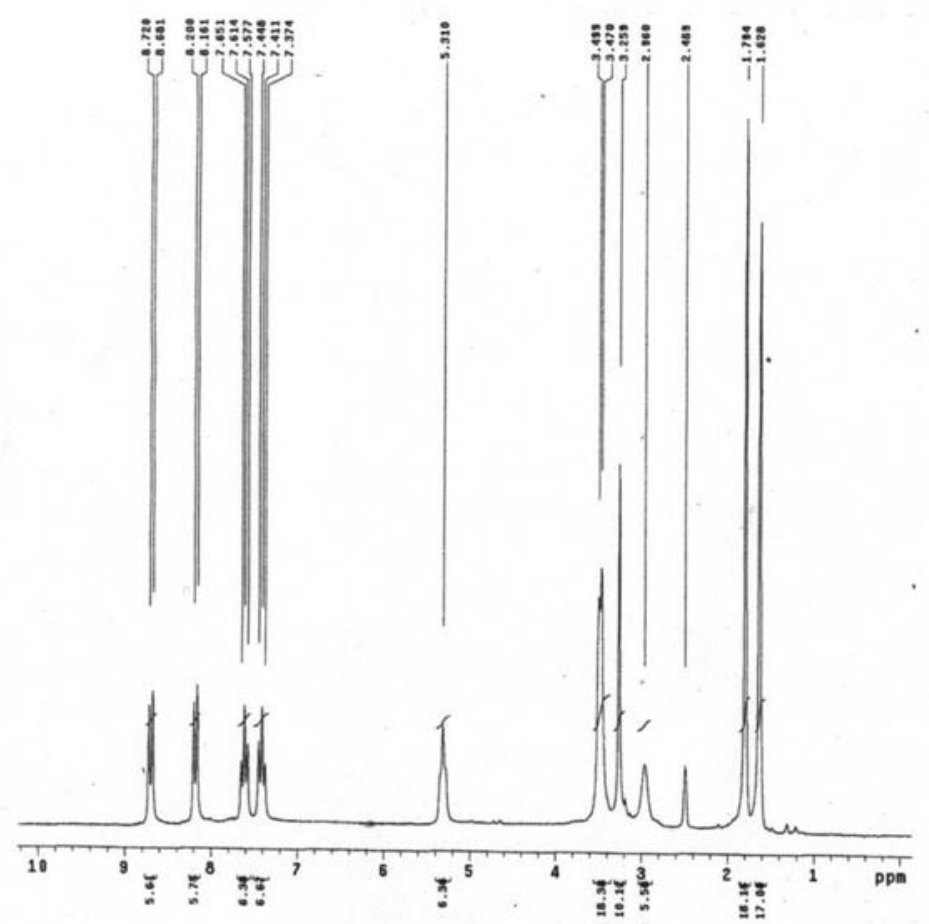

Figure S8. ${ }^{1} \mathrm{H}$ NMR spectrum (DMSO- $d_{6}, 200 \mathrm{MHz}$ ) of $\mathbf{2 b}$.

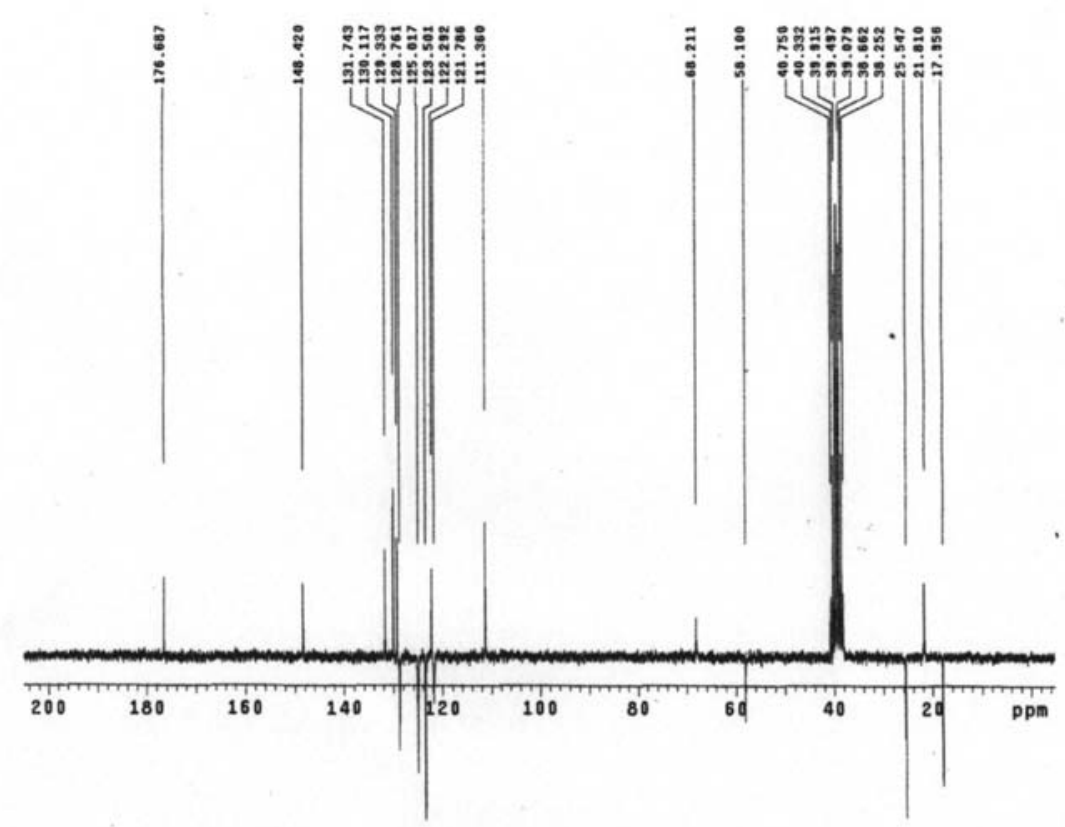

Figure S9. ${ }^{13} \mathrm{C}$ NMR (APT) spectrum (DMSO- $d_{6}, 50 \mathrm{MHz}$ ) of $\mathbf{2 b}$. 


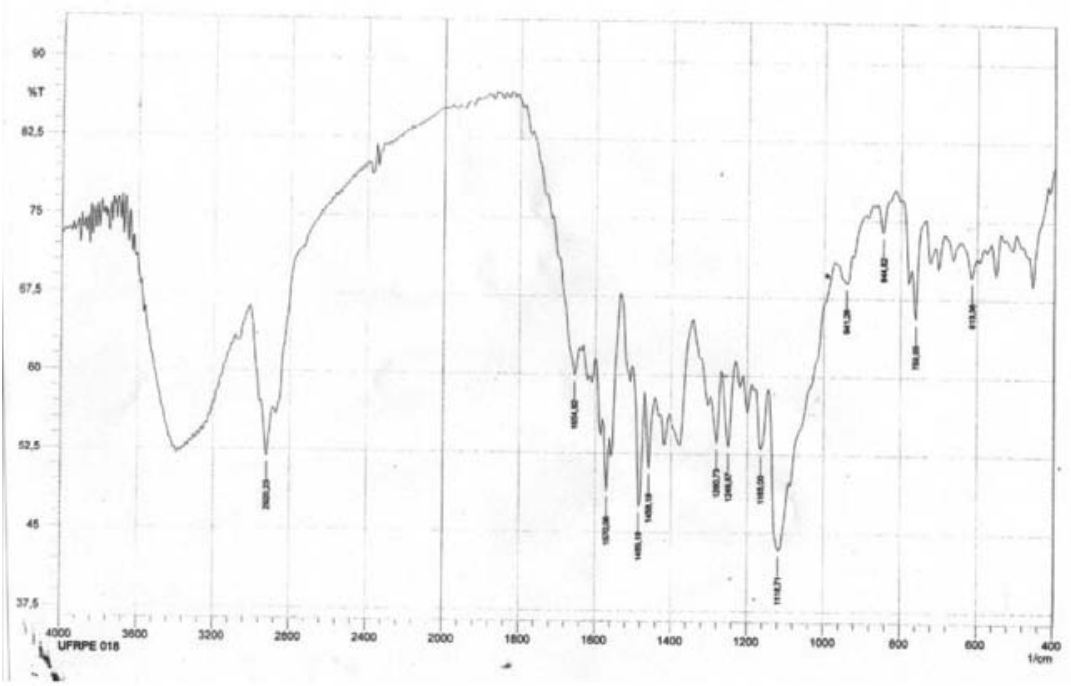

Figure S10. Infrared spectrum $\left(\mathrm{KBr}, \mathrm{cm}^{-1}\right)$ of $\mathbf{2 b}$.

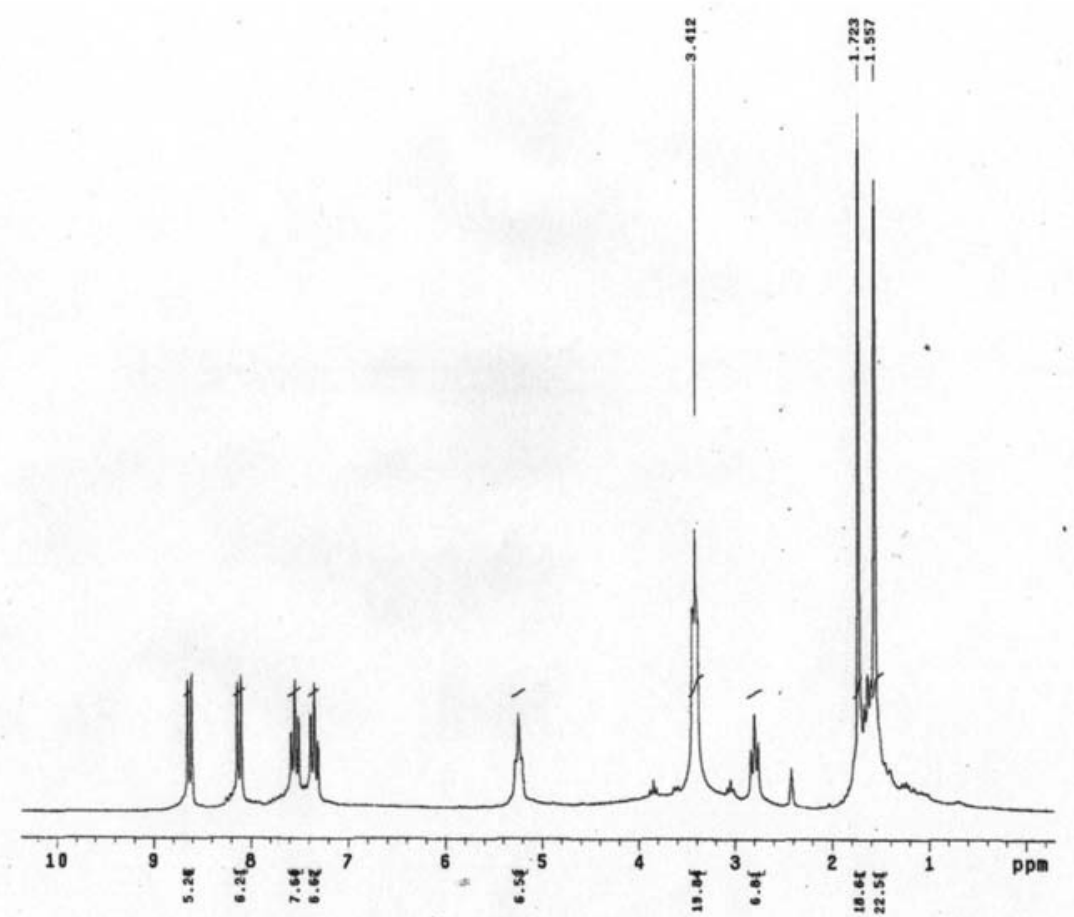

Figure S11. ${ }^{1} \mathrm{H}$ NMR spectrum (DMSO- $d_{6}, 200 \mathrm{MHz}$ ) of $\mathbf{2 c}$. 
Vol. 22, No. 4, 2011

Santos et al.

ST

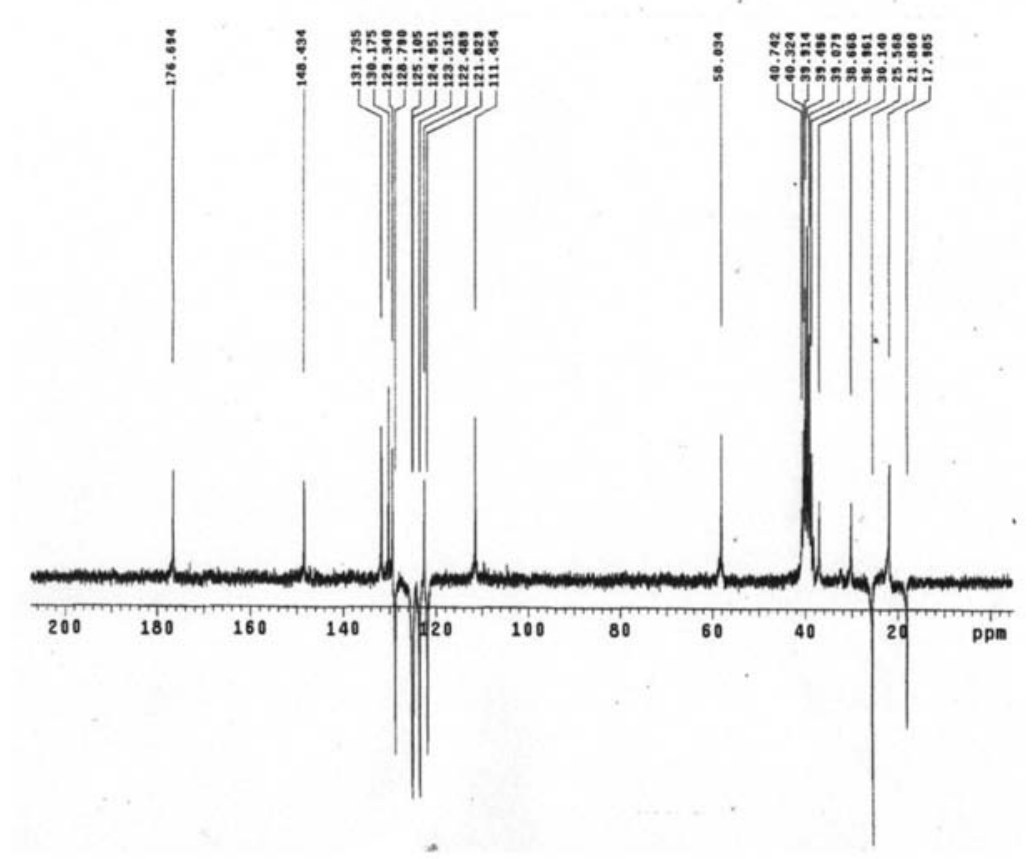

Figure S12. ${ }^{13} \mathrm{C}$ NMR (APT) spectrum (DMSO- $d_{6}, 50 \mathrm{MHz}$ ) of $\mathbf{2 c}$.

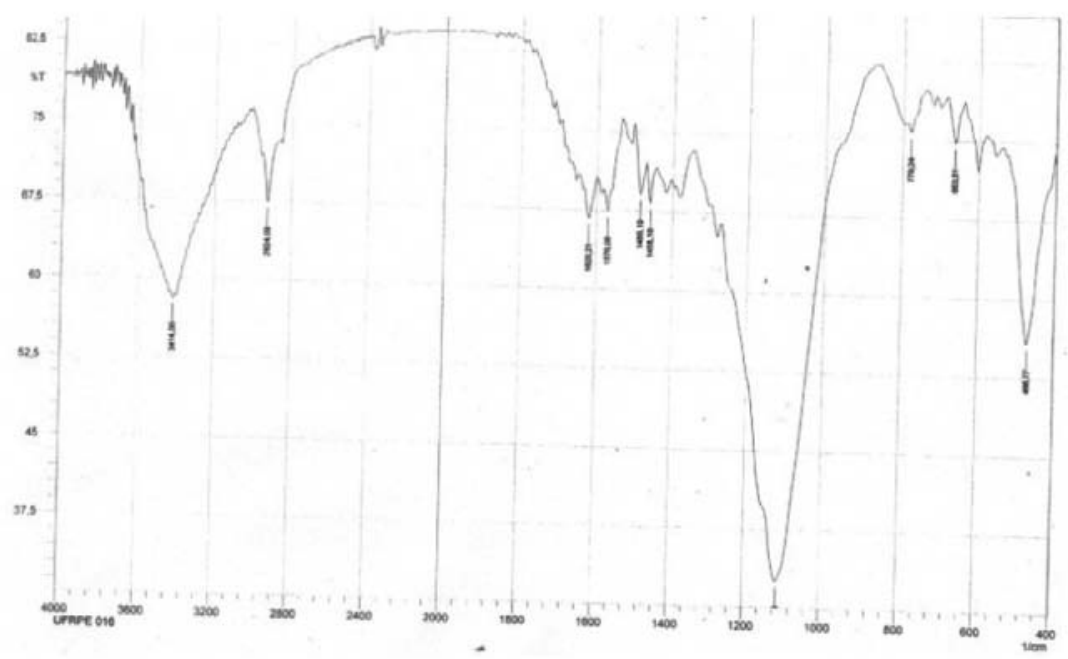

Figure S13. Infrared spectra $\left(\mathrm{KBr}, \mathrm{cm}^{-1}\right)$ of $\mathbf{2 c}$. 


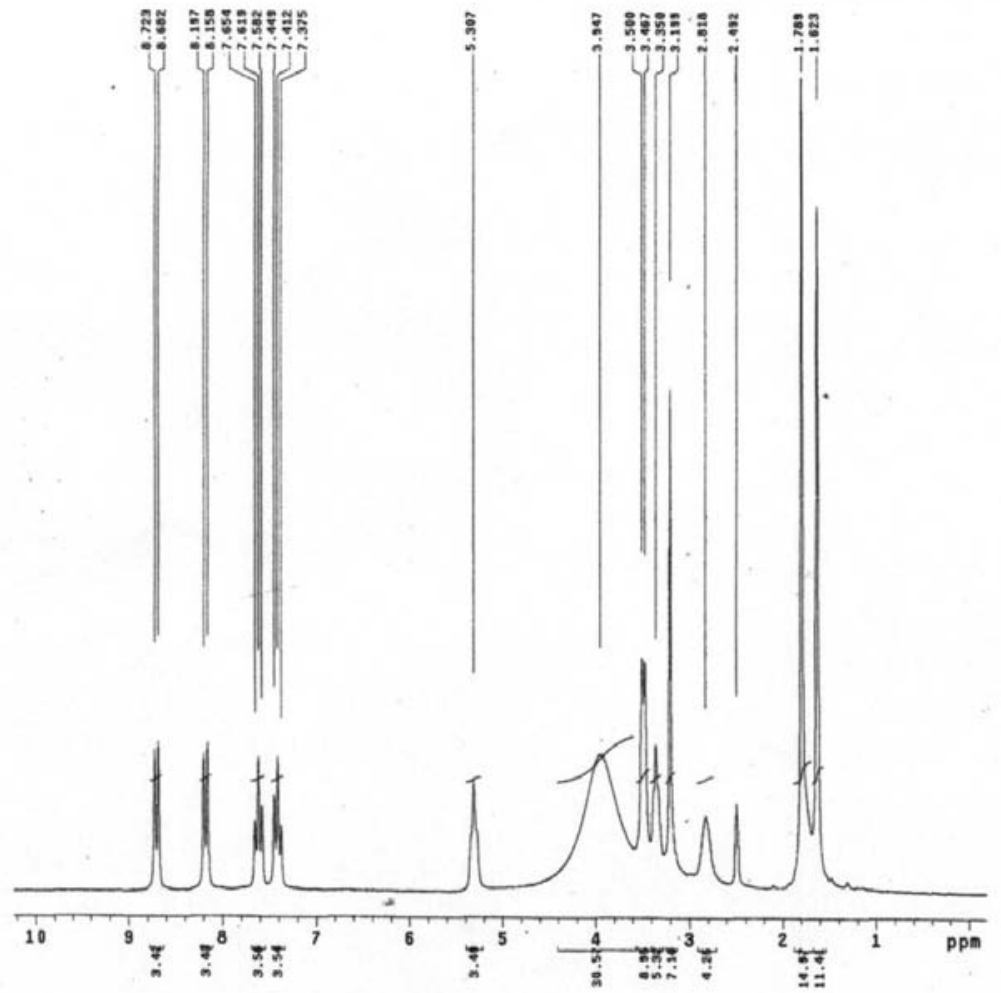

Figure S14. ${ }^{1} \mathrm{H}$ NMR spectrum (DMSO- $d_{6}, 200 \mathrm{MHz}$ ) of $\mathbf{2 d}$.

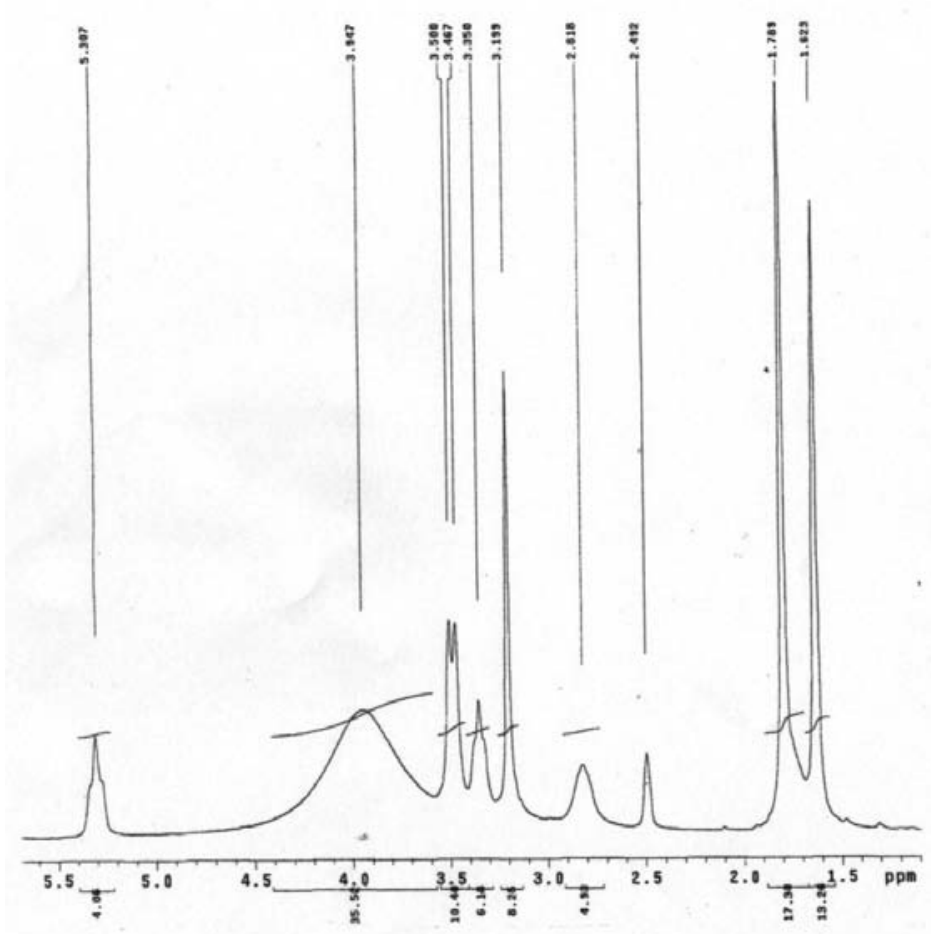

Figure S15. ${ }^{1} \mathrm{H}$ NMR spectrum expansion (DMSO- $d_{6}, 200 \mathrm{MHz}$ ) of $\mathbf{2 d}$. 
Vol. 22, No. 4, 2011

Santos et al.

Sq

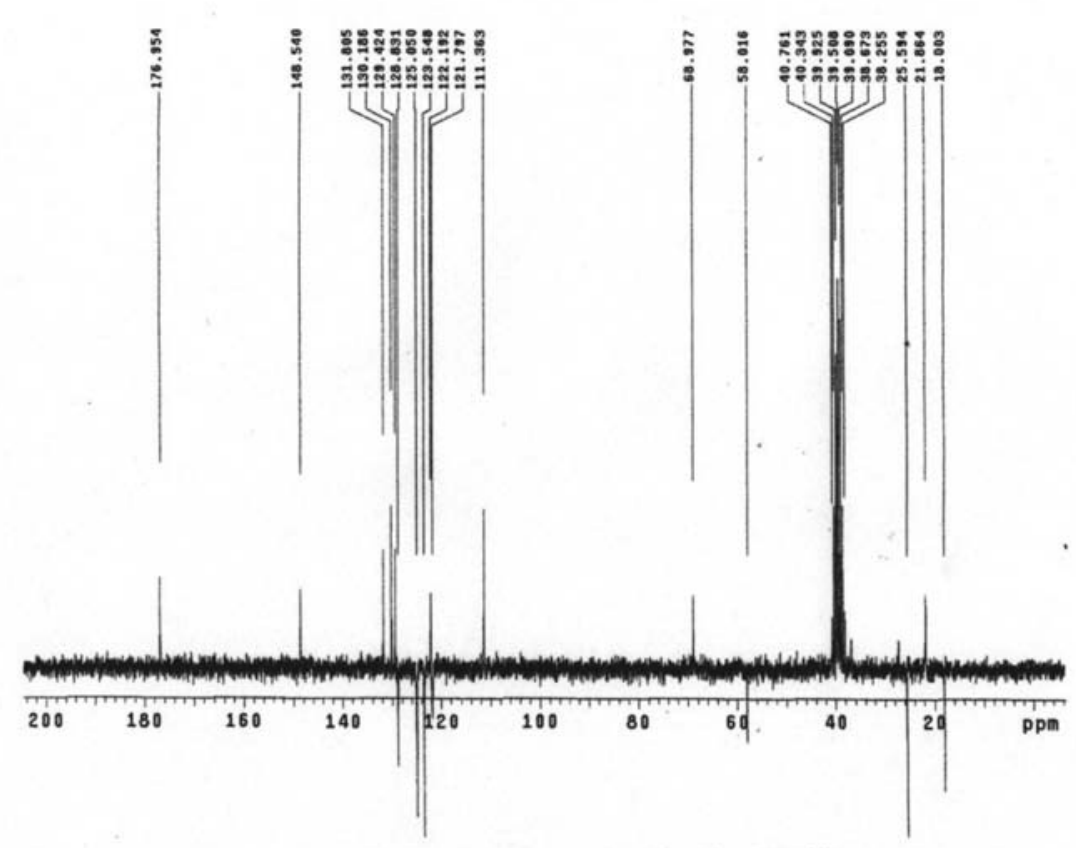

Figure S16. ${ }^{13} \mathrm{C}$ NMR (APT) spectrum (DMSO- $d_{6}, 50 \mathrm{MHz}$ ) of $\mathbf{2 d}$.

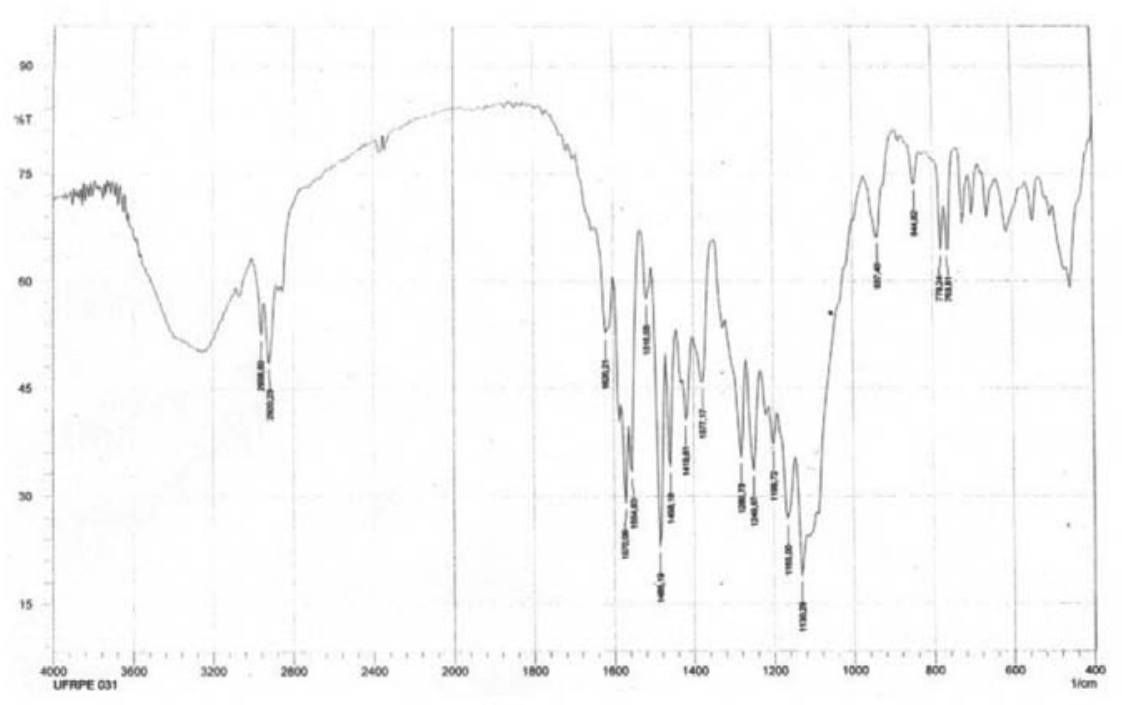

Figure S17. Infrared spectrum $\left(\mathrm{KBr}, \mathrm{cm}^{-1}\right)$ of $\mathbf{2 d}$. 


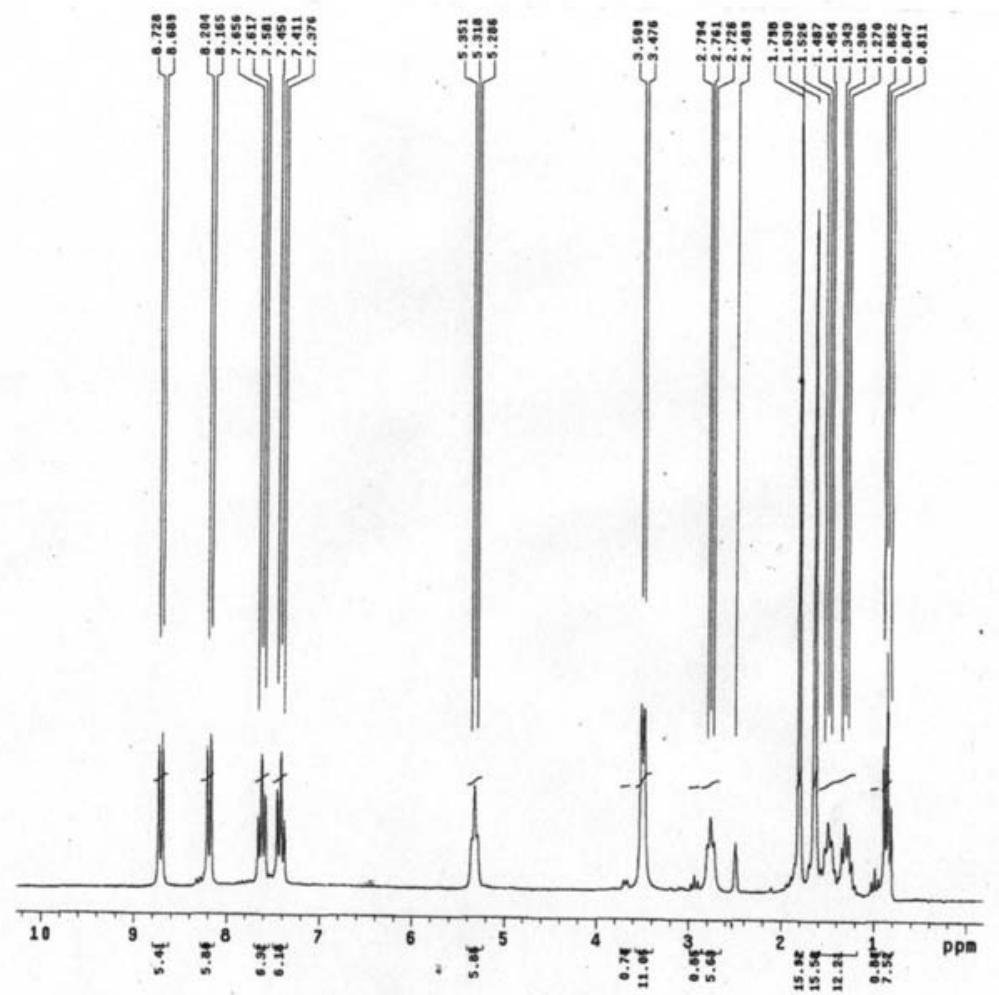

Figure S18. ${ }^{1} \mathrm{H}$ NMR spectrum (DMSO- $d_{6}, 200 \mathrm{MHz}$ ) of $2 \mathbf{e}$.

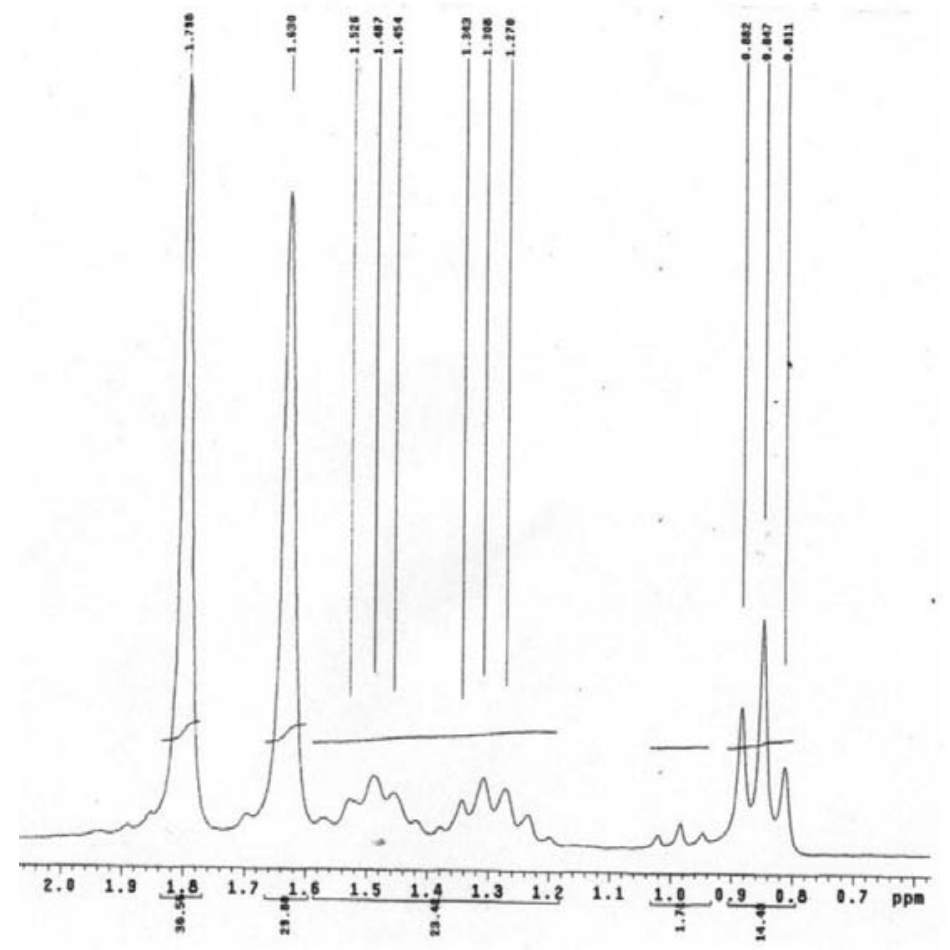

Figure S19. ${ }^{1} \mathrm{H}$ NMR spectrum expansion (DMSO- $d_{6}, 200 \mathrm{MHz}$ ) of $\mathbf{2 e}$. 


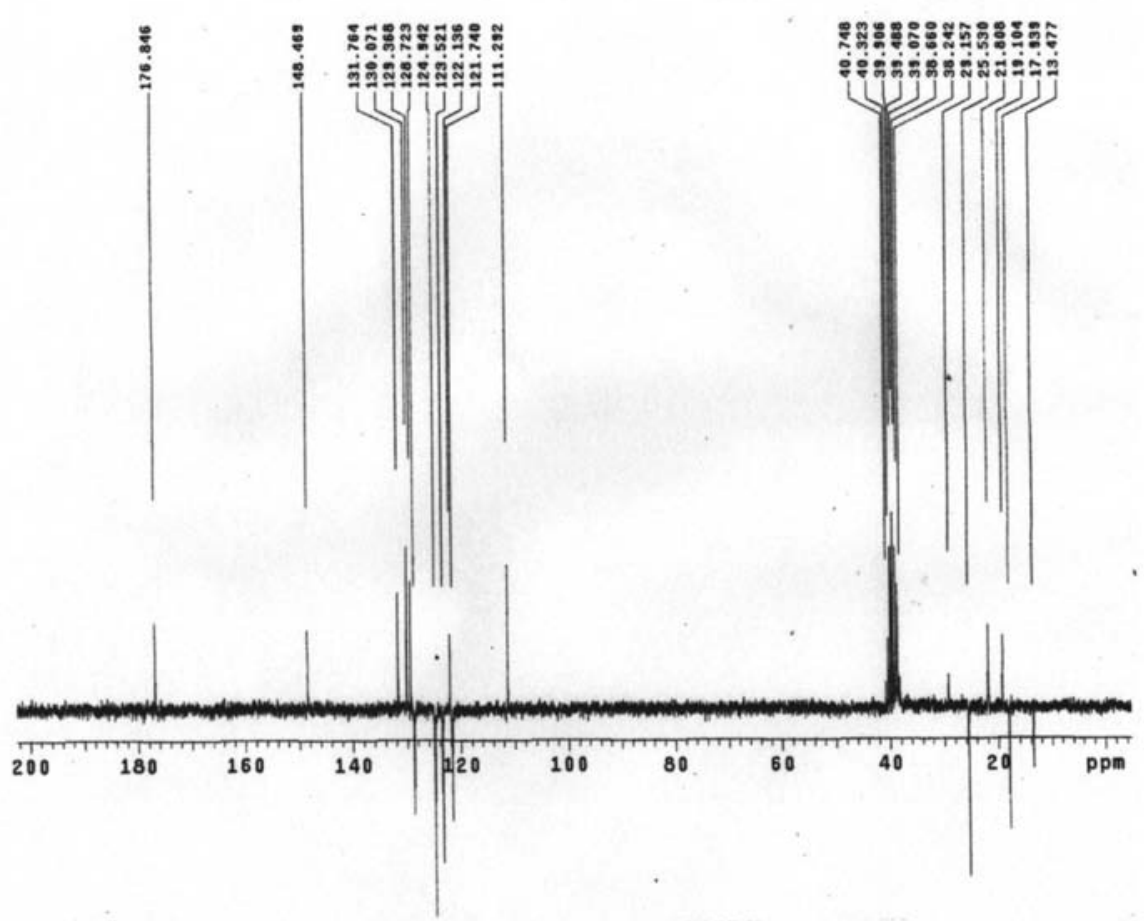

Figure S20. ${ }^{13} \mathrm{C}$ NMR (APT) spectrum (DMSO- $d_{6}, 50 \mathrm{MHz}$ ) of $\mathbf{2 e}$.

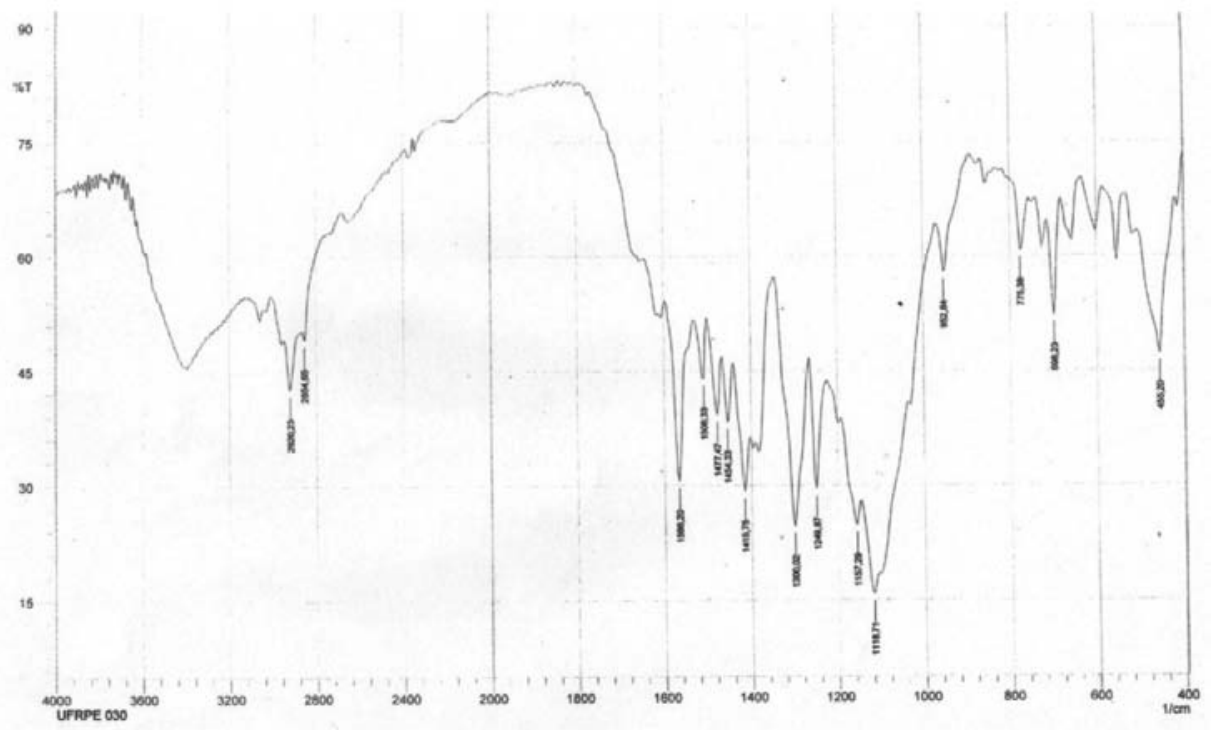

Figure S21. Infrared spectrum $\left(\mathrm{KBr}, \mathrm{cm}^{-1}\right)$ of $\mathbf{2 e}$. 


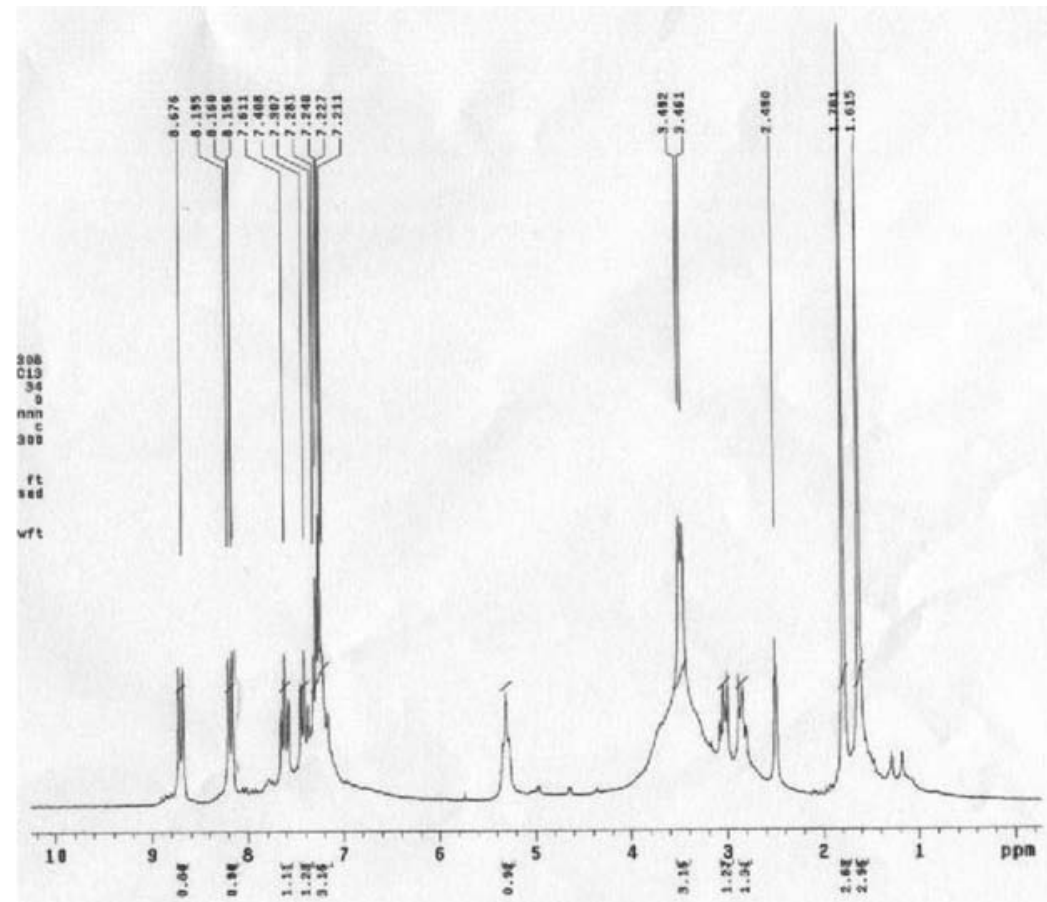

Figure S22. ${ }^{1} \mathrm{H}$ NMR spectrum (DMSO- $d_{6}, 200 \mathrm{MHz}$ ) of $\mathbf{2 f}$.

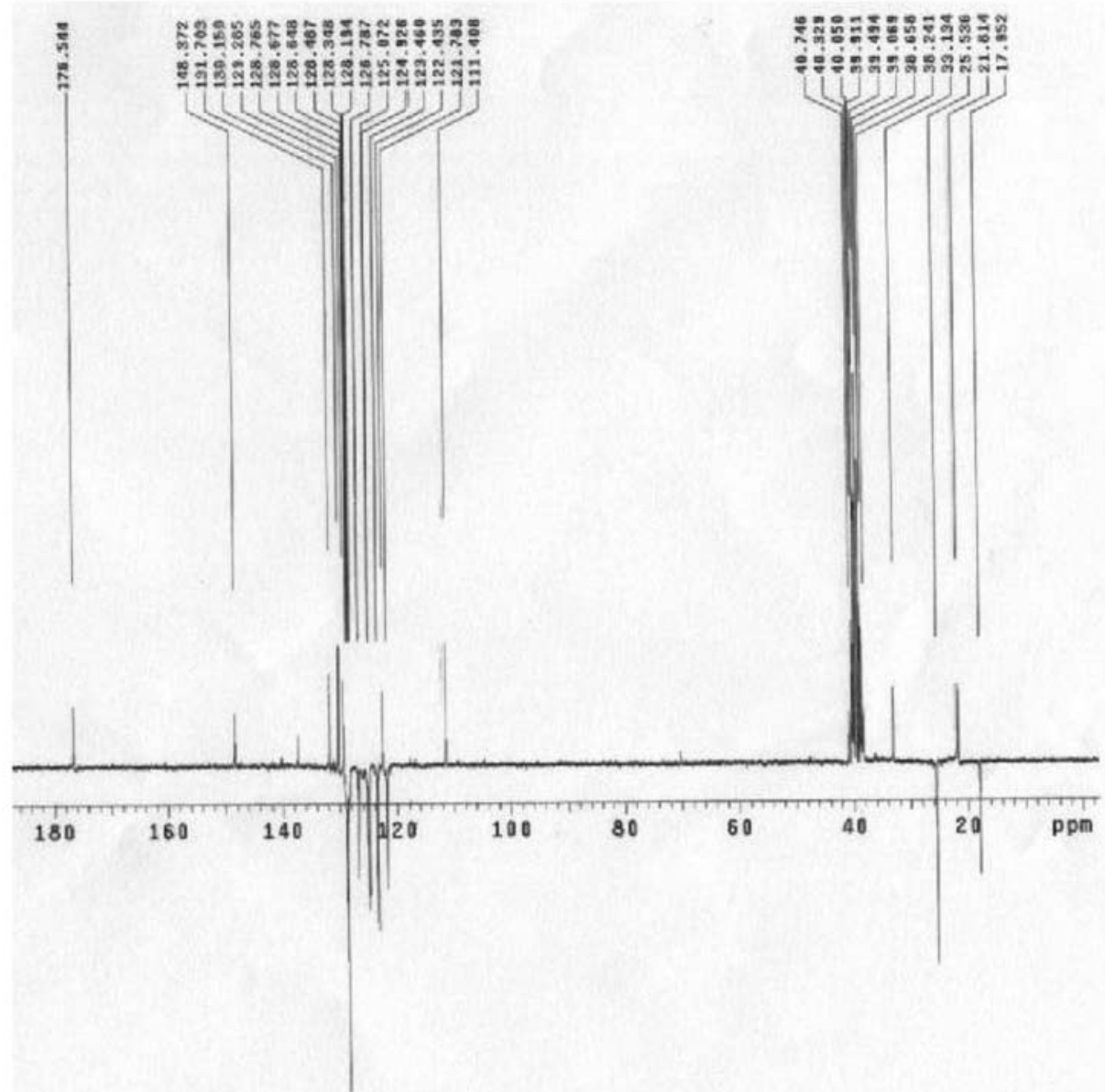

Figure S23. ${ }^{13} \mathrm{C}$ NMR (APT) spectrum (DMSO- $d_{6}, 50 \mathrm{MHz}$ ) of $2 \mathbf{f}$. 


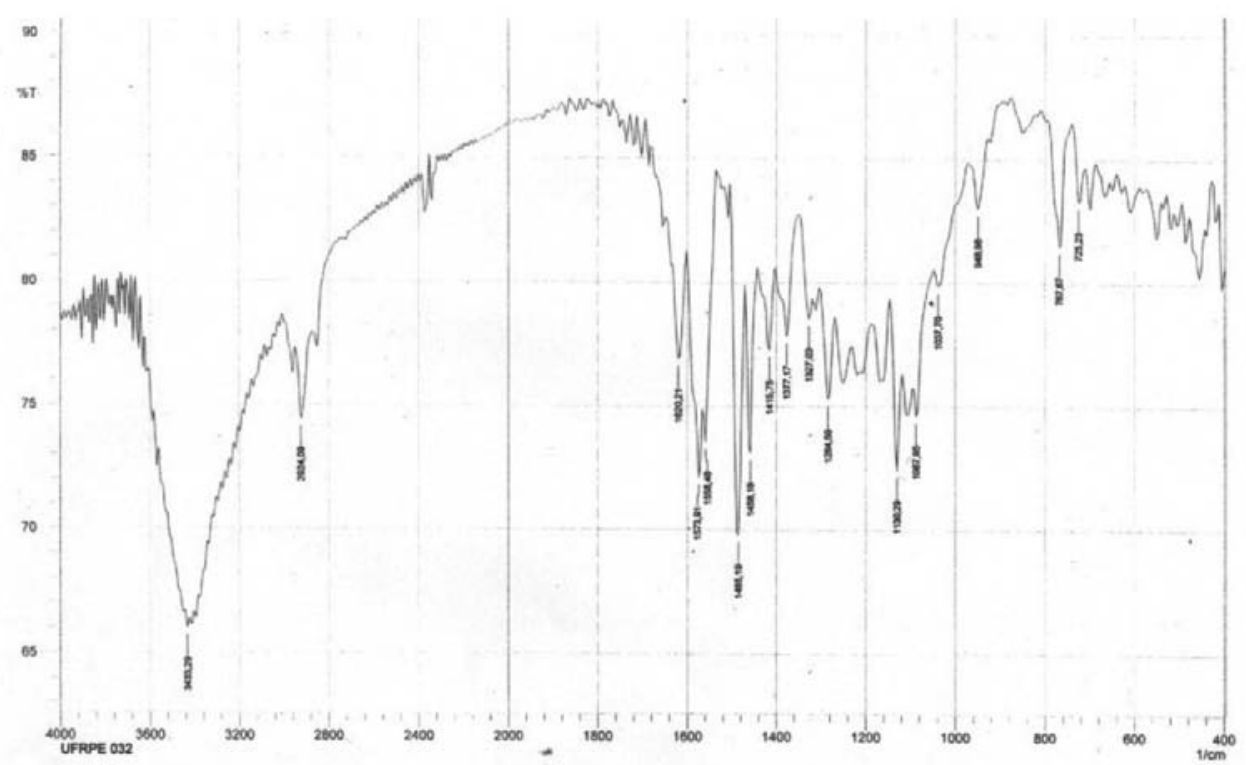

Figure S24. Infrared spectrum $\left(\mathrm{KBr}, \mathrm{cm}^{-1}\right)$ of $\mathbf{2 f}$.

\section{Fluorescence spectra (excit 590nm)}

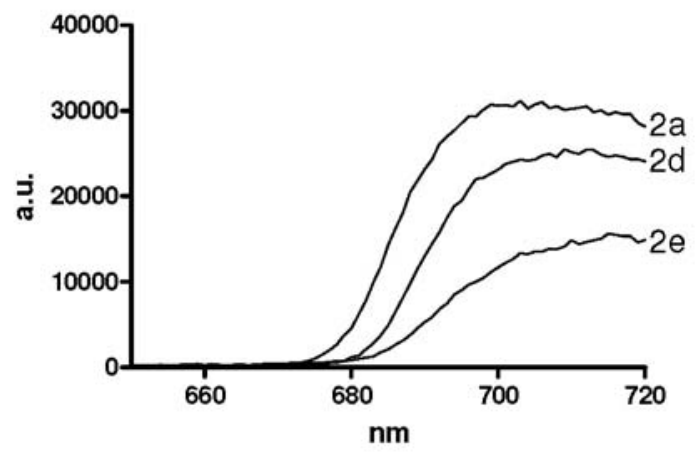

Figure S25. Fluorescence spectra (excit $590 \mathrm{~nm}$ ) of lapachol adducts (2). 